\title{
A Multi-Scale Model for Coupling Strands with Shear-Dependent Liquid
}

\author{
YUN (RAYMOND) FEI, Columbia University, USA \\ CHRISTOPHER BATTY, University of Waterloo, Canada \\ EITAN GRINSPUN and CHANGXI ZHENG, Columbia University, USA
}
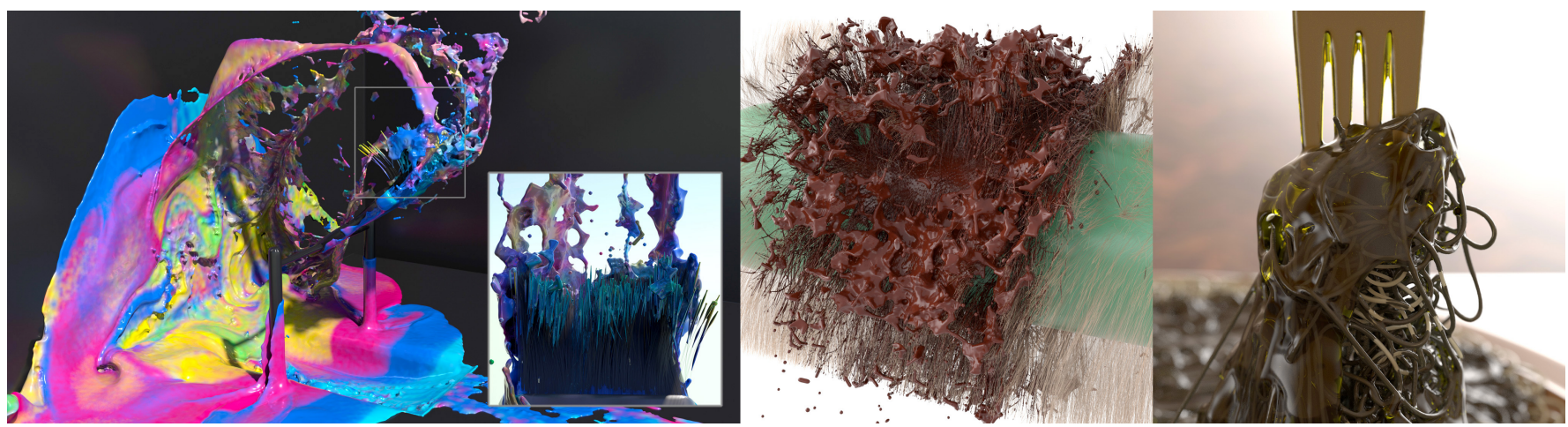

Fig. 1. Left: A rotating brush splashing through thick oil paint. The inset shows a zoomed view of paint on the bristles. Middle: Melted chocolate poured onto a hair-covered cylinder that rotates to mimic the shaking behavior of mammals. Right: Soba noodles covered with oyster sauce pulled upwards by a fork.

We propose a framework for simulating the complex dynamics of strands interacting with compressible, shear-dependent liquids, such as oil paint, mud, cream, melted chocolate, and pasta sauce. Our framework contains three main components: the strands modeled as discrete rods, the bulk liquid represented as a continuum (material point method), and a reduced-dimensional flow of liquid on the surface of the strands with detailed elastoviscoplastic behavior. These three components are tightly coupled together. To enable discrete strands interacting with continuum-based liquid, we develop models that account for the volume change of the liquid as it passes through strands and the momentum exchange between the strands and the liquid. We also develop an extended constraint-based collision handling method that supports cohesion between strands. Furthermore, we present a principled method to preserve the total momentum of a strand and its surface flow, as well as an analytic plastic flow approach for Herschel-Bulkley fluid that enables stable semi-implicit integration at larger time steps. We explore a series of challenging scenarios, involving splashing, shaking, and agitating the liquid which causes the strands to stick together and become entangled.

CCS Concepts: • Computing methodologies $\rightarrow$ Physical simulation

\section{ACM Reference Format:}

Yun (Raymond) Fei, Christopher Batty, Eitan Grinspun, and Changxi Zheng. 2019. A Multi-Scale Model for Coupling Strands with Shear-Dependent

Authors' addresses: Yun (Raymond) Fei, Columbia University, Computer Science, New York, NY, 10027, USA; Christopher Batty, University of Waterloo, Computer Science, Waterloo, ON, N2L 3G1, Canada; Eitan Grinspun; Changxi Zheng, Columbia University Computer Science, New York, NY, 10027, USA.

Permission to make digital or hard copies of all or part of this work for personal or classroom use is granted without fee provided that copies are not made or distributed for profit or commercial advantage and that copies bear this notice and the full citation on the first page. Copyrights for components of this work owned by others than the author(s) must be honored. Abstracting with credit is permitted. To copy otherwise, or republish, to post on servers or to redistribute to lists, requires prior specific permission and/or a fee. Request permissions from permissions@acm.org.

(C) 2019 Copyright held by the owner/author(s). Publication rights licensed to ACM.

0730-0301/2019/11-ART190 \$15.00

https://doi.org/10.1145/3355089.3356532
Liquid. ACM Trans. Graph. 38, 6, Article 190 (November 2019), 20 pages. https://doi.org/10.1145/3355089.3356532

\section{INTRODUCTION}

A brush stirring and spreading oil paint, shaving cream thrust onto a beard, and spaghetti tossed in tomato sauce all share a common trait. These scenarios are challenging to simulate due to the complex rheology of the liquid and the intricate interactions between the liquid and the strands.

In this work, we propose models and algorithms to capture the rich dynamic interactions between shear-dependent liquids and strands in scenarios such as these. A key element of what makes these situations unique is that shear-dependent liquids have strainand time-dependent viscosity. To give one example, the macroscopic behavior of shaving cream derives from the microscopic bubbles that it comprises. The stochastic rearrangement of the bubbles causes the liquid to exhibit shear-thinning effects, in which the continuum begins to flow more easily under large applied stress. In addition, shaving cream can undergo rate-dependent permanent deformations, a characteristic behavior of viscoplastic flows. These properties constrast starkly with Newtonian fluids in which viscous forces have a simple linear dependence on velocity.

Recent prior research on animating wet hair [Fei et al. 2017] and wet fabrics [Fei et al. 2018] has included models for the cohesion between strands, reduced-dimensional flow of water along a strand, and two-way coupling between liquid and submerged fabrics. These models, however, only account for Newtonian and incompressible liquids. Proper treatment of interactions with non-Newtonian and compressible liquids requires a fundamental reconsideration of the underlying model components and their interactions. 
For instance, when strands come into contact with a sheardependent liquid, a thin layer often adheres and flows along the strands. These layers, on the one hand, are generally too thin to be simulated by existing particle- or grid-based non-Newtonian fluid solvers; on the other hand, the

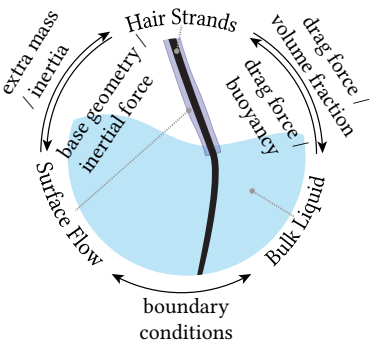
fluid can deform elastically or plastically, which is not captured by the prior reduced-dimensional surface water model. Therefore, a new method is necessary if we are to simulate these detailed shear-dependent flows economically.

Another influential effect is friction between wet strands, which is often a more significant factor in this context due to the influence of larger viscous and/or elastic forces. This fact suggests the need for an accurate, combined cohesive-frictional contact model to achieve realistic behavior.

Finally, the models used for the strands, the bulk liquid, and the reduced surface flow must be coupled together. We identify the following requirements for successful coupling: 1) Since the strands act as the base geometry of the surface flow, the surface flow should contribute extra mass and inertia to the strands. 2) The bulk liquid should exchange momentum with the submerged strands through drag forces and pressure gradients (buoyancy), which depend on the volume fraction of the submerged strands. 3) The surface flow and the bulk liquid should maintain consistent velocities and pressures where they meet, through appropriate boundary conditions. These requirements apply to both Newtonian and non-Newtonian fluids. However, some non-Newtonian fluids (e.g., shaving cream or pasta sauce) are compressible and can, therefore, change their volume during deformation, which leads to a more complicated calculation for pressure.

Our contribution in this paper is the development of a multiscale framework capturing the interactions between strands and compressible shear-dependent fluid, which includes:

- a reduced model for shear-dependent liquids flowing on the strands that accounts for elastic and plastic deformation (\$3.1);

- three-way coupling between discrete elastic rods, continuum bulk liquid, and reduced surface flow in a principled framework, which accounts for a wide range of rheologies and degrees of compressibility $(\S 3.4)$;

- a modified second-order Coulomb cone model for stable cohesive and frictional effects when strands collide (§3.5); and

- a stable semi-implicit solver for shear-dependent fluid, with a more efficient semi-analytical formulation of plastic flow $(\S 4)$.

\section{RELATED WORK}

\subsection{Strand Simulation}

Physics Model for Strands. There have been decades of effort toward simulating hair strands. For an overview, we refer the reader to several thorough reviews [Bertails et al. 2008; Hadap et al. 2007; Ward et al. 2007]. Popular models for single-strand dynamics include mass-spring models [Petrovic et al. 2005; Selle et al. 2008], various
Cosserat rod models [Grégoire and Schömer 2007; Spillmann and Teschner 2007], the Super-Helix model [Bertails et al. 2006], and the discrete elastic rod (DER) model [Bergou et al. 2010, 2008; Kaldor et al. 2010; Kaufman et al. 2014]. Since some strands (e.g., human hairs) are stiff and often do not exhibit significant stretching, a number of efficient constraint-based models have also recently been developed [Deul et al. 2018; Han and Harada 2013; Kugelstadt and Schömer 2016; Müller et al. 2012; Soler et al. 2018]. In this paper, although our method is not limited to a specific strand model, we choose the DER model for its efficiency and accuracy, which has also been exploited in mechanics and robotics research [Jawed et al. 2018].

Collision and Cohesion between Wet Hairs. Prior work on wet hair [Fei et al. 2017; Lin 2014, 2015] modeled cohesion and contact as a penalty-like force. However, the penalty model is known to have multiple issues. First, it requires a finely-tuned stiffness parameter to balance collision and cohesion effects, and thereby avoid both instability and tunneling. Second, handling friction accurately tends to be difficult for penalty models. To avoid these issues, we propose to model cohesive and repulsive effects together as constraints. Prior work on rod-contact using constraints [Bertails-Descoubes et al. 2011; Daviet et al. 2011] can avoid tunneling as long as the detection phase captures all contacts and the solver converges. More recent approaches [Gornowicz and Borac 2015; Kaufman et al. 2014] improved stability using nonlinear Newton solves. In this paper, we adopt these improvements to non-smooth contact dynamics and further extend the method to handle cohesive effects. Wet hair also tends to have a higher friction coefficient than dry hair [Bhushan et al. 2005]; our constraint-based method allows us to handle such wet frictional effects easily.

Although uncommon in computer animation, the use of nonsmooth contact dynamics to handle cohesion is widely adopted in mechanics for simulating cracks [Jean et al. 2001; Kadau et al. 2002]. In these works, the second-order Coulomb cone is displaced in the normal direction to achieve cohesive effects. To prevent the re-cohesion of cracked parts, they introduced hysteresis on the cohesion coefficient. These methods partly inspire our work on collision handling for cohesive wet strands.

Also, a few authors have treated strands or other deformable thin structures as a fluid-like material and hence adopted continuumbased collision handling [Fei et al. 2018; Guo et al. 2018; Hadap and Magnenat-Thalmann 2001; Jiang et al. 2017; Lee et al. 2019; McAdams et al. 2009], where contact and friction are solved globally on a uniform grid. These methods either need an extra pass of traditional penalty-based collision handling or can be numerically difficult for simulating stiff strands. With a constraint-based solver, however, the contacts are solved iteratively and then the impulses are integrated on each strand individually, which can be much more efficient especially when each strand has a limited number of vertices and collisions ${ }^{1}$.

\footnotetext{
${ }^{1}$ In all examples presented in this paper, we have at most 80 vertices on a single strand, which is typical in strand simulation (e.g., [Kaufman et al. 2014]).
} 


\subsection{Simulating Liquid with Complex Rheology}

Recently, hybrid grid/particle-based methods [Fu et al. 2017; Hu et al. 2018; Jiang et al. 2015; Zhu and Bridson 2005] for simulating liquid have become popular due to their visual plausibility and efficacy for pressure computation. A complete review of the basics of this method for fluid simulation in computer graphics can be found in the book by Bridson [2015]. The particle-in-cell method from fluid dynamics was extended to handle general elastoviscoplastic materials, leading to the material point method (MPM) [Jiang et al. 2016; Sulsky et al. 1994]. Models based on MPM have been extensively used in computer animation to simulate various materials and phenomena [Daviet and Bertails-Descoubes 2016; Gaume et al. 2018; Hu et al. 2018; Klár et al. 2016; Nagasawa et al. 2019; Ram et al. 2015; Stomakhin et al. 2013, 2014; Wolper et al. 2019]. More recently, variants of MPM have been proposed to increase the computational efficiency [Fang et al. 2019; Gao et al. 2017, 2018b; $\mathrm{Hu}$ et al. 2018]. One recent approach coupled MPM with the discrete element method to deliver more detailed dynamics [Yue et al. 2018] at modest cost. In our work, we adopt the moving-least-squares MPM (MLS-MPM) [Hu et al. 2018] as our discretization scheme for its efficiency, and couple this MPM with discrete elastic rods. To avoid nullspace issues during the pressure solve, we adapt MLS-MPM to a staggered grid, similar to the work of Stomakhin et al. [2014].

The family of smoothed particle hydrodynamics (SPH) methods is another common alternative for fluid simulation [Ihmsen et al 2014; Monaghan 1994; Müller et al. 2003]. Although SPH can also be used to simulate liquids with complex rheology [Yang et al. 2017], our use of a grid simplifies momentum exchange between the liquid and strands, especially when both phases need to be integrated (semi-)implicitly for better stability.

To handle complex fluids, we need a constitutive model that is both accurate and efficient. In this paper, we adopt the constitutive model from Yue et al. [2015], which targets Herschel-Bulkley fluids [1926] with von Mises yield condition [1913], and employs a f2-flow model for plasticity [Simo 1988]. This model covers a wide range of materials such as mud, cream, chocolate, and pasta sauce. In prior work [Simo 1988; Yue et al. 2015], the stress computed from this model was integrated explicitly. However, this leads to tiny time steps that are at odds with the larger time steps that are possible with DER. Therefore, we develop an analytical formulation of plastic flow for Herschel-Bulkley fluids, which enables semi-implicit integration of the stress and dramatically increases the size of a stable time step.

Depth-averaged flows. For centuries, researchers in fluid dynamics have considered models for depth-averaged flows [2009; 1995], such as shallow water equations (or Saint-Venant equations [1871]) for inertial fluid on planar boundaries, and lubrication theory [Oron et al. 1997] for viscous fluids. These depth-averaged models are also extensively used in computer animation [Azencot et al. 2015; Hinsinger et al. 2002; Kass and Miller 1990; Vantzos et al. 2017, 2018; Wang et al. 2007]. Recently, Fei et al. [2017] developed a reduceddimensional water model for (strictly Newtonian) liquid flowing on individual wet hairs.
Research on depth-averaged models for non-Newtonian liquid is relatively sparse [Hassine 2013; Saramito and Wachs 2017]. Recently, Ionescu and colleagues extended the shallow water model to handle general viscoplastic liquids [Ionescu 2010, 2013a,b; Ionescu and Lupaşcu 2016; Ionescu et al. 2015]. Prior work, however, did not consider the elastic deformation of the liquid. We draw inspiration from these approaches to derive a depth-averaged model for elastoviscoplastic liquid on strands, where the liquid deforms purely elastically before yielding and deforms plastically afterward.

\subsection{Theory of Mixtures}

A complete review of early work in mixture theory, as well as the history of research in the area can be found in the book by de Boer [2012]. Most of the theory of mixtures has focused on coupling granular material with a Newtonian, incompressible fluid [Bedford and Drumheller 1983]. Among the few exceptions, Pritchett [1978] derived the equations for coupling solid with compressible fluid, which was revisited and validated with better numerical treatments by Kuipers et al. [1992]. Later, theories about mixtures of (visco)elastic materials were developed by Iesan [1994; 2008]; a thorough review can be found in the survey by Pearson and Tardy [2002]. Following these prior works, the shear stresses of the fluid and the strands are independently applied to the liquid and strands, and the momentum between the two phases is exchanged through drag force.

In computer animation, mixtures containing fluid have been treated differently depending on the fluid's Reynolds number. Some approaches focus on Darcy flow [1856], where the fluid creeps slowly. These works deal with diffusion inside a solid material [Lenaerts et al. 2008; Lin 2014, 2015; Patkar and Chaudhuri 2013; Rungjiratananon et al. 2012, 2008]. Mixtures with higher Reynolds number fluids were first studied in computer animation by Nielsen and Østerby [2013]. Subsequently, SPH-based methods have been widely adopted to handle multi-fluid or multi-phase flow phenomena [Ren et al. 2014; Yan et al. 2016; Yang et al. 2017, 2015]. These methods exchange momentum between different phases through a viscous term between particles. Researchers have also adopted mixture theory [Bandara and Soga 2015] to simulate porous sand or fabrics mixed with water [Fei et al. 2018; Gao et al. 2018a; Tampubolon et al. 2017]. In these works, the authors treated both the solid and liquid phases as continua. This treatment differs from our setting, in which the strands are simulated as discrete elements.

As part of the interaction forces, the drag effect mainly depends on the relative velocity between the liquid and solid, as shown by experimental studies (e.g., [Acharya et al. 1976]), based on which various drag models have been developed. One treats both the liquid and solid as continuous media [Fei et al. 2018, 2017; Tampubolon et al. 2017] and the other treats the solid as a collection of discrete elements [Gao et al. 2018a; Zhou et al. 2010]. Because of the discrete nature of strands, the drag force model we need falls into the latter category.

Drag Force between Two Continuums. Traditional mixture theory treats both the liquid and solid as continuous phases [Anderson and Jackson 1967]; drag models under this scheme have a long history [Carman 1937; Ergun 1952; Forchheimer 1901]. Recently, Fei 
et al. [2018] adopted a modern, unified drag formulation [Yazdchi and Luding 2012] to couple fabrics with Newtonian liquid. Since we couple a discrete phase with a continuous phase, however, these drag models for two continuums are not appropriate.

Drag Force between Continuum and Discrete Elements. Instead, we must integrate the drag forces over discrete elements and couple them with the continuous phase, which leads us to the CFD-DEM method [Tsuji et al. 1993]. Due to its wide applicability to fluidization and pneumatic conveying, the discrete element method (DEM) has been increasingly popular for the simulation of particles immersed in flowing fluids. In contrast to classical mixture theory that treats both liquid and solid as continua, the CFD-DEM method simulates solids as discrete elements, and couples them with liquid through homogenization [Zhong et al. 2016; Zhou et al. 2010].

Drag forces used for CFD-DEM are most often formulated for a single discrete element. These drag forces, however, also differ widely in terms of the particular drag models and drag coefficients. Among various drag models, Di Felice's is widespread and reasonably general [1994]; it also suits our needs since it is compatible with different drag coefficients and fits experimental data for both spherical and non-spherical particles [Hilton et al. 2010], as well as for either Newtonian or non-Newtonian fluids [Li and Zhao 2018] In this work, we adopt Di Felice's formula to compute drag between strands and liquid.

The accuracy of the drag force, finally, depends on choosing the drag coefficient. Among the abundant literatures [Zhong et al. 2016], we focus on drag coefficients applicable to complex fluid and irregular particles. An extensive survey of these studies is presented in a book by Chhabra [2006]. In the present work, we adopt the model proposed by Mauret and Renaud [1997; 2004] since it has been extensively validated against physical experiments [Atapattu et al. 1995; Beaulne and Mitsoulis 1997; Beris et al. 1985; Rajitha et al. 2006; Tabuteau et al. 2007].

\section{PHYSICAL MODELS}

We begin by presenting three primary physical models to support simulating strands interacting with non-Newtonian liquids: i) a reduced non-Newtonian surface flow model, ii) a discrete strand model driven by strand-liquid interaction, and iii) a model for bulk liquid mixed with submerged strands. In describing the three physical models, our notation will be to use a subscript $\tau$ to indicate a quantity related to the surface flow (e.g., $u_{\tau}$ for surface flow velocity), a subscript $\mathrm{s}$ for the strand's quantities, and a subscript $\mathrm{f}$ for the bulk liquid's quantities.

\subsection{Non-Newtonian Strand Surface Flow}

The first component of our work is a model for non-Newtonian liquid flowing on the surface of a strand. While potentially imperceptible on a single thin strand, surface flow can significantly change the collective dynamics (and thus appearance) of many strands interacting and agglomerating together (Fig. 1). Unfortunately, capturing the shallow depth of such a surface flow with a grid-based fluid solver would require an excessively refined background grid, making them a poor choice in this case. One recent approach simulated a shallow water-like flow on strands [Fei et al. 2017], but accounted

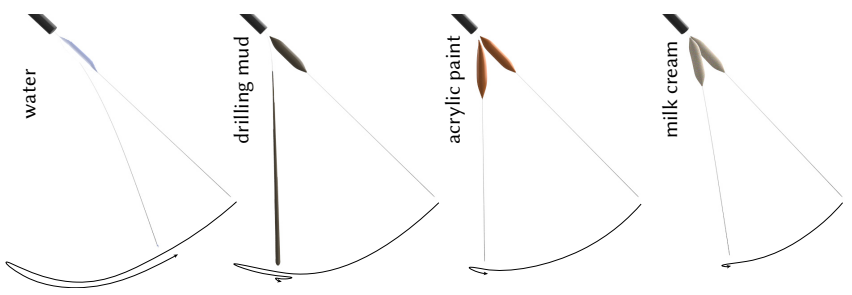

Fig. 2. Surface flows of various materials. Different material properties yield drastically different behaviors. The beginning and ending frames are shown overlaid, with the curved arrows indicating the motion of strands. Please check our supplemental video for the actual animation.

only for inviscid and non-Newtonian liquids. A new non-Newtonian reduced surface flow model is needed.

3.1.1 Reduced Surface Flow Kinematics. To present our reduced model, we start with surface flow kinematics described using cylindrical coordinate frames. These coordinate frames are aligned along the strand's centerline. In these frames, the surface liquid velocity is denoted as $\boldsymbol{u}_{\tau}=\left(u_{\tau}, v_{\tau}, \omega y\right)^{T}$, where $u_{\tau}$ and $v_{\tau}$ are liquid velocities along the centerline's tangential and radial directions, respectively, and $\omega$ is the angular velocity around the strand (see Fig. 3 for an illustration of these notations). Our reduced surface flow model is established with a few assumptions on the velocity field:

i) The flow thickness $h_{\tau}$ and strand radius $r$ are much smaller than the length $L$ of the strand. Thus, we only need to consider the average longitudinal velocity $u_{\tau}$ across its depth and orientation, and the average velocity $u_{\tau}$ is invariant in $y-$ and $\theta-$ coordinates (i.e., $\frac{\partial u_{\tau}}{\partial y}=0$ and $\frac{\partial u_{\tau}}{\partial \theta}=0$ ).

ii) The bulk modulus of a non-Newtonian fluid is often several orders of magnitude larger than its shear modulus ${ }^{2}$. Given the surface flow layer is also thin, consequently, the volumetric change of the surface flow is negligible in comparison to its translation and shear motion. In other words, assuming the surface flow to be isochoric (incompressible) will not affect its visual appearance: $\nabla \cdot \boldsymbol{u}_{\tau}=0$ and $\operatorname{det} \mathbf{F}=1$, where $\mathbf{F}$ is the deformation gradient.

iii) Because the strand radius is small and the surface flow layer is thin, the fluid has relatively strong surface tension $-O\left(\left(h_{\tau}+\right.\right.$ $r)^{-1}$ ) according to the Young-Laplace equation [1805] - that keeps the fluid uniform around the strand centerline. Therefore, we ignore the angular motion of the surface flow (i.e., we assume $\omega=0)$.

Assumptions (i) and (iii) ensure that the surface liquid has no strain in the angular direction: its $3 \mathrm{D}$ strain tensor is reduced to a $2 \mathrm{D}$ strain tensor-a 2D symmetric matrix having three independent elements. The two diagonal elements indicate the normal strains separately in the longitudinal and radial directions, while the offdiagonal element involves both directions. We refer to supplemental $\S S 1$ for a brief review of 2D non-Newtonian fluid simulation.

The 2D strain tensor can be reduced further. Since the strand's radius $r$ is much smaller than its length $L$, the surface flow behavior

\footnotetext{
${ }^{2}$ For example, shaving cream has a bulk modulus $1.09 \mathrm{e} 6 \mathrm{dyne} / \mathrm{cm}^{2}$ while its shear modulus is $2.9 \mathrm{e} 3 \mathrm{dyne} / \mathrm{cm}^{2}$. Similar materials (e.g., pasta sauce) or materials that have higher bulk but lower shear modulus (e.g., paint and mud) are used in this paper. See the supplemental §S14 for detailed parameters.
} 


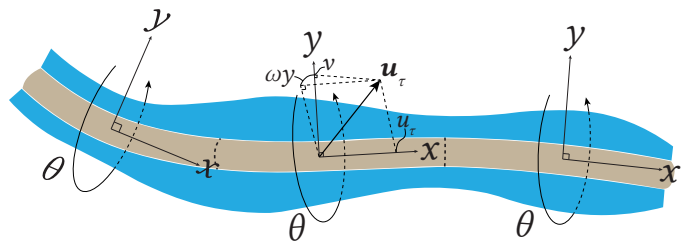

Fig. 3. Coordinate system defined along the strand centerline. At each point of the strand's centerline, the $x$-axis, i.e., longitudinal axis, of the frame is along the centerline's tangential direction; the $y$-axis, i.e., radial axis, is the strand's radial direction; and the $\theta$-axis is the angular direction around the strand. The local velocity field (in the cross-section at each point) of the non-Newtonian surface fluid is $\boldsymbol{u}_{\tau}=\left(u_{\tau}, v_{\tau}, \omega y\right)^{T}$, where $u_{\tau}$ and $v_{\tau}$ are fluid velocities along the centerline's tangential and radial directions, respectively, and $\omega$ is the angular velocity around the strand.

in the strand cross-section differs from that in the longitudinal direction by a scaling factor $\epsilon \equiv r / L$ where $\epsilon \ll 1$. Through a multiscale asymptotic analysis with respect to $\epsilon$ (see details in supplemental $\S S 2$ ), we find that the off-diagonal element of the strain tensor has only a second-order (i.e., $\epsilon^{2}$ ) contribution to the deviatoric part of the strain, which is the strain component determining the shear stress and plastic flow. This observation suggests that we can ignore the off-diagonal element.

Moreover, due to incompressibility (i.e., assumption (ii) above), the strains in the longitudinal and radial directions are related: compression in one direction leads to an expansion in the other. Based on this condition and the derivation in supplemental §S2, we further reduce the $2 \mathrm{D}$ strain to a single scalar, which we call the reduced Cauchy-Green strain,

$$
c_{\tau}^{E} \equiv b_{x x}-b_{y y},
$$

where $b_{x x}$ and $b_{y y}$ are the principal strains in the longitudinal and radial directions (i.e., the two diagonal elements).

\subsubsection{Reduced Surface Flow Dynamics.}

Momentum equation. Incorporating this reduced strain leads to a modified form of the momentum equation for non-Newtonian fluids. As derived in supplemental §S2, the standard 3D NavierStokes momentum equation becomes a simpler 1D equation,

$$
A_{\tau} \rho_{\mathrm{f}} \frac{\mathrm{D}_{u_{\tau}} u_{\tau}}{\mathrm{D} t}=A_{\tau} f_{\mathrm{ext}, x}+\frac{\partial}{\partial x}\left(\mu A_{\tau} c_{\tau}^{E}\right)-C u_{\tau}
$$

where $\mu$ is the fluid's shear modulus, $\rho_{\mathrm{f}}$ is the liquid density, $A=$ $\pi h_{\tau}\left(h_{\tau}+2 r\right)$ is the annular cross-sectional area of the flow with a thickness $h_{\tau}$, and $f_{\text {ext, } x}$ indicates external force density in the tangential direction. The external forces include gravity, inertial force due to strand motion [Fei et al. 2017], as well as the coupling forces among the surface flow, strands, and bulk fluid, to be discussed in $§ 3.4$. The last term $C u_{\tau}$ in (2) is the friction force, which we will discuss shortly.

The reduced strain $c_{\tau}^{E}$ is not only used to model the shear force in (2), but is also time-varying and obeys a flow rule. For nonNewtonian fluids, the strain rate is determined by both elastic deformation and plastic flow. Their specific contributions, emerging from our multiscale asymptotic analysis of the deviatoric strain given in

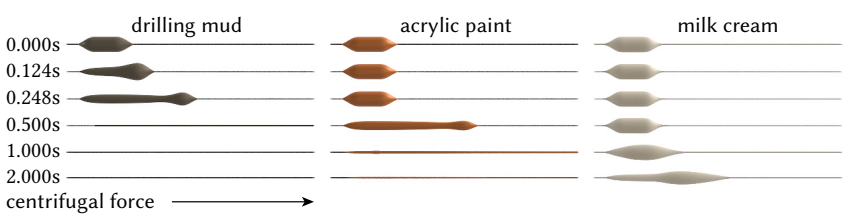

Fig. 4. Surface flows under increasing centrifugal force. Three strands with surface flows of different materials are rotated with increasing angular velocity over time to apply increasing centrifugal force. The camera is aligned with the strands' tangential direction for legibility. Compared with the mud, the paint and cream only yield and start flowing under sufficiently high stress, where the cream has especially large yield stress (1200dyne $/ \mathrm{cm}^{2}$ ).

supplemental §S2, are given by

$$
\frac{\mathrm{D}_{u_{\tau}} c_{\tau}^{E}}{\mathrm{D} t}=\underbrace{2 \frac{\partial u_{\tau}}{\partial x} \sqrt{c_{\tau}^{E^{2}}+4}}_{\text {elastic deformation }}-\underbrace{\sqrt{2} \gamma\left(s_{\tau}\right)\left(c_{\tau}^{E}+\sqrt{c_{\tau}^{E^{2}}+4}\right) \operatorname{sgn}\left(c_{\tau}^{E}\right)}_{\text {plastic flow }},
$$

where $s_{\tau} \equiv \frac{1}{\sqrt{2}} \mu\left|c_{\tau}^{E}\right|$ is the magnitude of the shear stress, and $\gamma(s)$ is the flow rate function. In this work, we choose to use the flow rate function in the Herschel-Bulkley model [Herschel and Bulkley 1926], an effective phenomenological model for a wide range of viscoplastic materials such as cream [Yue et al. 2015], mud [Kelessidis et al. 2006], paint [Bochkarev et al. 2009], chocolate [Ardakani et al. 2014], and pasta sauce [Nagasawa et al. 2019]. A brief introduction to the Herschel-Bulkley model is provided in supplemental §S1, where the specific form of the flow rate function is given in (S13).

Friction between surface flow and strand. Because of its viscosity, the non-Newtonian surface flow experiences friction on the strand surface. Microscopically, the friction is caused by intermolecular interactions that prevent the viscous liquid from either separating from or slipping along the solid surface [Sochi 2011]. We adapt a widely used model of this viscosity-induced friction that assumes a linear relationship between the slip velocity and the friction on the surface - the Navier boundary condition [Lamb 1993]. Concretely, the friction force can be modeled by the $C u_{\tau}$ term in (2), where, according to [Gerbeau and Perthame 2001], the coefficient $C$ is related to the surface flow thickness $h_{\tau}$ by

$$
C=A_{\tau} \kappa\left(h_{\tau}+\frac{\kappa h_{\tau}^{2}}{3 \tilde{\eta}}\right)^{-1} .
$$

Here, $\kappa$, defined as $\kappa \equiv \tilde{\eta} / b$, is the friction coefficient depending on the effective fluid viscosity $\tilde{\eta}$ [Andreini et al. 2012], and the Navier slip length, $b$. The latter is a constant depending on the specific fluid and solid materials [Brochard and De Gennes 1992; Pit et al. 1999]. This model, albeit simple, can closely match experimental results for various non-Newtonian fluids [Schowalter 1988].

When using the Herschel-Bulkley model for non-Newtonian fluid, we can derive its effective viscosity using the generalized Newtonian fluid model [Irgens 2014] and obtain

$$
\tilde{\eta}=\sqrt{\frac{2}{3}} \tau_{Y}\left(\frac{\left|u_{\tau}\right|}{h_{\tau}}\right)^{-1}+\eta\left(\frac{\left|u_{\tau}\right|}{h_{\tau}}\right)^{n-1},
$$

where $\tau_{Y}$ is the yield stress, $\eta$ is the flow consistency index, and $n$ is the flow behavior index. With the effective viscosity estimated, 
we use (4) in combination with the strand's annular cross-sectional area $A_{\tau}$ to obtain the formula for $C$ in our surface flow momentum equation (2):

$$
C=\pi \frac{h_{\tau}+2 r}{b+h_{\tau} / 3} \tilde{\eta} .
$$

Mass conservation. Lastly, mass conservation for the surface flow follows prior work [Fei et al. 2017]:

$$
\frac{\mathrm{D}_{u_{\tau}} A_{\tau}}{\mathrm{D} t}+A_{\tau} \frac{\partial u_{\tau}}{\partial x}=0 \text {. }
$$

In summary, our non-Newtonian reduced surface flow model consists of a momentum equation (2), a mass conservation law (7), a time evolution equation for the reduced Cauchy-Green strain (3), as well as the Herschel-Bulkley flow rate function (i.e., (S13) in supplemental §S1).

\subsection{Strand Dynamics}

A strand's motion is heavily influenced by the liquid layer flowing on its surface. If the liquid were simply fixed to the strand surface (without any relative motion), then its only effect would be to cause the effective mass of the strand to become the sum of the strand mass, $m_{\mathrm{s}}$, and the liquid mass, $m_{\tau}$. However, surface flow motion relative to the strand will induce an additional inertial force on the strand.

This intuition can be formalized by writing out the total inertia of the strand and its surface fluid. We model a strand as a discrete elastic rod [Bergou et al. 2008], and use $\boldsymbol{u}_{\mathrm{s}}$ to denote the strand velocity at a discrete strand vertex. The surface fluid flows along the strand with a velocity $u_{\tau}$ relative to the strand (recall §3.1). Then, the absolute velocity of the surface flow is $\boldsymbol{u}_{\mathrm{s}}+\boldsymbol{t} u_{\tau}$, where $\boldsymbol{t}$ is the tangential direction of the strand. The total inertia is

$$
m_{\mathrm{s}} \frac{\mathrm{D}_{\boldsymbol{u}_{\mathrm{s}}} \boldsymbol{u}_{\mathrm{s}}}{\mathrm{D} t}+m_{\tau} \frac{\mathrm{D}_{\boldsymbol{u}_{\mathrm{s}}+\boldsymbol{t} u_{\tau} \boldsymbol{u}_{\mathrm{s}}}}{\mathrm{D} t}=\left(m_{\mathrm{s}}+m_{\tau}\right) \frac{\mathrm{D}_{\boldsymbol{u}_{\mathrm{s}}} \boldsymbol{u}_{\mathrm{s}}}{\mathrm{D} t}+m_{\tau} u_{\tau} \nabla_{\tau} \boldsymbol{u}_{\mathrm{s}}
$$

The right hand side expands and regroups terms on the left, revealing the extra inertial force as the last term. With this total inertia, the momentum equation for the strands is

$$
\tilde{m}_{s} \frac{\mathrm{D}_{\boldsymbol{u}_{\mathrm{s}}} \boldsymbol{u}_{\mathrm{s}}}{\mathrm{D} t}=f_{\text {int }, s}+f_{\mathrm{ext}, \mathrm{s}}+\tilde{m}_{s} \boldsymbol{g}-m_{\tau} \boldsymbol{u}_{\tau} \nabla_{\tau} \boldsymbol{u}_{\mathrm{s}},
$$

where the effective mass $\tilde{m}_{s} \equiv m_{\mathrm{S}}+m_{\tau}, \boldsymbol{g}$ is the gravitational constant, and the last term comes from that of (8). The term $f_{\text {int, } s}$ represents the internal forces of the discrete elastic rod, including bending, twisting (see [Bergou et al. 2008] for their details), and stretching force (alternatively, replaced with an inextensible projection [Müller et al. 2012] for better stability). The external force, $f_{\text {ext,s }}$, includes strand-strand (and strand-solid) contact forces as well as the coupling forces with the bulk fluid. These forces will be elaborated in $\S 3.4$.

Remark. Prior work by Fei et al. [2017] for simulating strandwater interactions also considers the inertial force contribution by surface water flows. However, in their model, the extra inertial force is explicitly added at the end of each timestep. By contrast, we derived this force term in a more principled manner and incorporated it into the momentum equation, allowing for implicit integration (see §4). In supplemental §S5, we show that, when using an explicit integration, the momentum transport presented by Fei et al. [2017]

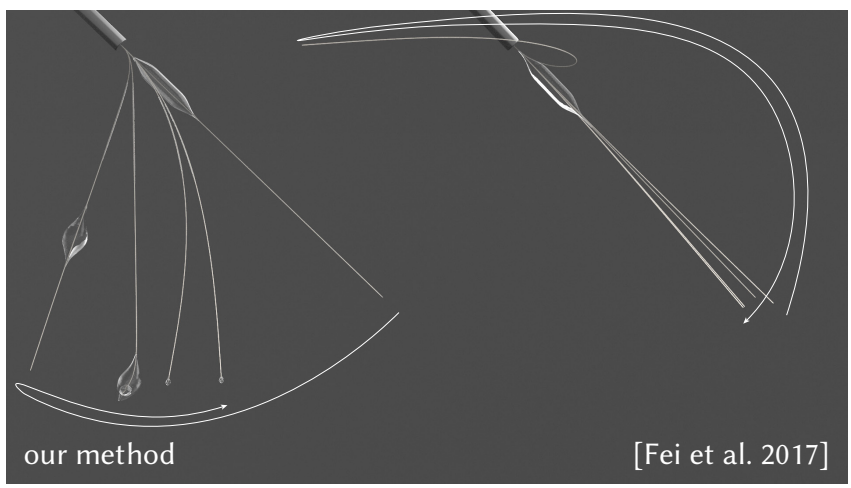

Fig. 5. Comparison with Fei et al. [2017]. A heavy droplet (tetrachloroethylene with mass density $1.622 \mathrm{~g} / \mathrm{cm}^{3}$ ) flows on a strand. Left: With our principled momentum equation for the coupled flow, the extra momentum of strand caused by the motion of surface flow can be stably integrated. Right: The explicit modification to the strand momentum proposed by Fei et al. [2017] causes instability.

is equivalent to solving (9) with its rightmost term. In other words, both the formulations make sense; however, our formulation enables implicit integration. As demonstrated in Fig. 5, explicitly adding the extra inertial force (as in [Fei et al. 2017]) impairs simulation stability, whereas our implicit integration approach remains quite stable.

\subsection{Bulk Liquid in a Mixture}

An effective way of modeling bulk non-Newtonian liquid is to treat it as a continuum, as most non-Newtonian simulation methods have done [Irgens 2008]. In our scenarios, the bulk liquid will inevitably interact with submerged strands, which, as discussed in $\$ 3.2$, are modeled as discrete rod elements. Thus we are confronted with two contradictory simulation approaches. We must somehow reconcile these disparate views if we are to enforce mass and momentum conservation for both the liquid and the submerged strands in a unified framework.

A natural idea is to homogenize the discrete elements (i.e., strands in our case) and treat the combination of liquid mixed with discrete elements as a porous medium, whose behavior can be described using classic mixture theory [Anderson and Jackson 1967; Borja 2006]. Indeed, the CFD-DEM method [Zhong et al. 2016; Zhou et al. 2010] is based on this premise, but focuses on incompressible Newtonian fluids [Gao et al. 2018a; Tampubolon et al. 2017]. Here we extend this methodology to the simulation of compressible, non-Newtonian fluids.

3.3.1 Volume Fraction. We begin by homogenizing the volume of the strands. Suppose that the $i$-th strand element has a volume $V_{i}$. Then, in a porous medium where strands are mixed with liquid, the local volume fraction of the strand at any point $\boldsymbol{x}$ can be estimated using a kernel-weighted average [Gao et al. 2018a]:

$$
\epsilon_{\mathrm{s}}(\boldsymbol{x})=\frac{1}{V^{*}} \sum_{i} w_{R, i}(\boldsymbol{x}) V_{i}
$$

Here the summation is taken over a small region of the continuum; in practice we use one grid cell. The total volume of the region is denoted $V^{*}$, and $w_{R, i}(\boldsymbol{x})$ is the kernel function centered at the $i$-th 
element with a kernel radius $R$. Similar to the prior work [Fei et al. 2018; Gao et al. 2018a], we use a quadratic B-spline as $w_{R}$.

We can homogenize the velocity of the discrete strand elements in a similar fashion. Let $\boldsymbol{u}_{\mathrm{s}, \mathrm{i}}$ denote the velocity of the $i$-th strand element. The homogenized strand velocity $\overline{\boldsymbol{u}}_{\mathrm{s}}(\boldsymbol{x})$ at position $\boldsymbol{x}$ is defined as

$$
\epsilon_{\mathrm{s}}(\boldsymbol{x}) \overline{\boldsymbol{u}}_{\mathrm{s}}(\boldsymbol{x})=\frac{1}{V^{*}} \sum_{i} w_{R, i}(\boldsymbol{x}) V_{i} \boldsymbol{u}_{\mathrm{s}, i} .
$$

The strand volume fraction evolves over time as the liquid and strand move relative to one another. As we derive in detail in supplemental §S3, the material derivative of the strand volume fraction can be expressed as

$$
\frac{\mathrm{D}_{\boldsymbol{u}_{\mathrm{f}}} \epsilon_{\mathrm{S}}(\boldsymbol{x})}{\mathrm{D} t}=\frac{1}{V^{*}} \sum_{i} V_{i} \nabla w_{R, i} \cdot\left(\boldsymbol{u}_{\mathrm{f}}\left(\boldsymbol{x}_{i}\right)-\boldsymbol{u}_{\mathrm{s}, i}\right),
$$

where $\boldsymbol{u}_{\mathrm{f}}\left(\boldsymbol{x}_{i}\right)$ is the liquid velocity around the strand's $i$-th element position, $\boldsymbol{x}_{i}$.

Lastly, since the volume is a mixture of strands and liquid, the volume fraction of the liquid is related to that of the strands via $\epsilon_{\mathrm{f}}=1-\epsilon_{\mathrm{s}}$, and its material derivative is $\mathrm{D}_{\boldsymbol{u}_{\mathrm{f}}} \epsilon_{\mathrm{f}}(\boldsymbol{x}) / \mathrm{D} t=-\mathrm{D}_{\boldsymbol{u}_{\mathrm{f}}} \epsilon_{\mathrm{S}}(\boldsymbol{x}) / \mathrm{D} t$ This material derivative is needed for the mixture's mass conservation law-which we will present in §3.3.3-because a change of volume fraction at position $\boldsymbol{x}$ leads to a change of the mixture's local effective density at $\boldsymbol{x}$.

Remark. In the derivation of (12), we assume that each strand element is incompressible: it can be stretched, bent, or twisted, but always preserves its volume. This assumption is justified by the fact that strands are often thin and stiff. We note that Selle et al. [2008] also treated strands collectively as a continuum and assumed incompressible motion. The key difference of our approach is that we only assume each individual strand element is incompressible. The collective volume of strands can still disperse or contract as needed.

3.3.2 Momentum Equation. Using the notion of volume fraction and following classic mixture theory [Pritchett 1978], we now have the momentum equation for bulk liquid:

$$
\epsilon_{\mathrm{f}} \rho_{\mathrm{f}} \frac{\mathrm{D}_{\boldsymbol{u}_{\mathrm{f}}} \boldsymbol{u}_{\mathrm{f}}}{\mathrm{D} t}=\epsilon_{\mathrm{f}} \nabla \cdot \boldsymbol{\sigma}+f_{\mathrm{ext}, \mathrm{f}}+\epsilon_{\mathrm{f}} \rho_{\mathrm{f}} \boldsymbol{g},
$$

where $\sigma$ is the liquid's internal stress due to its volumetric stress (i.e., pressure $p$ ) and shear stress, namely,

$$
\boldsymbol{\sigma}=-p \mathbf{I}_{3}+\mu J^{-2 / 3} \operatorname{dev}\left[\mathbf{b}^{\mathrm{E}}\right]
$$

Here $\mu$ is the liquid's shear modulus, $\mathbf{I}_{3}$ is the $3 \times 3$ identity matrix, the deviatoric operator $\operatorname{dev}[\cdot]$ is defined as $\operatorname{dev}[\boldsymbol{x}] \equiv \boldsymbol{x}-(\operatorname{tr}[\boldsymbol{x}] / 3) \mathbf{I}_{3}$, the left Cauchy-Green elastic strain tensor is denoted $\mathbf{b}^{\mathrm{E}}$, and $J$ is the determinant of the liquid's deformation gradient (see details in supplemental §S1).

The term $f_{\text {ext,f }}$ in Eq. (13) represents the external forces applied on the liquid, including the drag force produced by relative motion between the liquid and submerged strands, and interaction forces between the bulk liquid and surface flow on the strands. Both forces will be elaborated in $\S 3.4$.

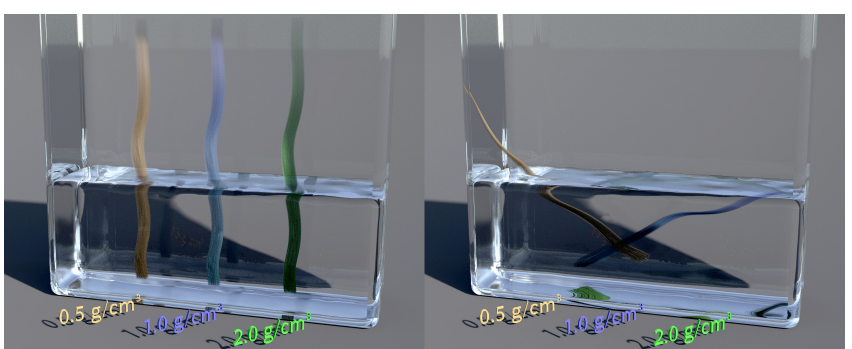

Fig. 6. Buoyancy-left: $0.0 \mathrm{~s}$, right: $4.0 \mathrm{~s}$. With the pressure gradient computed using our method, we can correctly handle the buoyancy of strands in water (mass density $1.0 \mathrm{~g} / \mathrm{cm}^{3}$ ), where the light brown strands (mass density $0.5 \mathrm{~g} / \mathrm{cm}^{3}$ ) float, neutrally buoyant blue strands (mass density $1.0 \mathrm{~g} / \mathrm{cm}^{3}$ ) drift, and heavy green strands (mass density $2.0 \mathrm{~g} / \mathrm{cm}^{3}$ ) sink.

3.3.3 Mass Conservation. According to mixture theory [Kuipers et al. 1992; Pritchett 1978], the mass conservation law for bulk liquid in the mixture is

$$
\frac{\mathrm{D}_{\boldsymbol{u}_{\mathrm{f}}} \epsilon_{\mathrm{f}} \rho_{\mathrm{f}}}{\mathrm{D} t}+\epsilon_{\mathrm{f}} \rho_{\mathrm{f}} \nabla \cdot \boldsymbol{u}_{\mathrm{f}}=0
$$

where $\rho_{\mathrm{f}}$ is the non-Newtonian liquid's density and $\epsilon_{\mathrm{f}} \rho_{\mathrm{f}}$ is the liquid's effective density in the mixture. Both $\rho_{\mathrm{f}}$ and $\epsilon_{\mathrm{f}}$ are spatially varying, though for brevity we do not indicate their dependence on $\boldsymbol{x}$ in (15). Many non-Newtonian materials (such as milk cream) are compressible, and therefore $\rho_{\mathrm{f}}(\boldsymbol{x})$ depends on the determinant $J(\boldsymbol{x})$ of the liquid's deformation gradient, which measures how much an infinitesimal liquid region has compressed or expanded-that is, $J(\boldsymbol{x})=\rho_{\mathrm{f}, 0} / \rho_{\mathrm{f}}(\boldsymbol{x})$, where $\rho_{\mathrm{f}, 0}$ is the liquid's (rest) material density. Using $J$ to express $\rho_{\mathrm{f}}$ in (15), we obtain the material derivative of $J$,

$$
\frac{\mathrm{D}_{\boldsymbol{u}_{\mathrm{f}} J}}{\mathrm{D} t}=J\left(\epsilon_{\mathrm{f}}^{-1} \frac{\mathrm{D}_{\boldsymbol{u}_{\mathrm{f}}} \epsilon_{\mathrm{f}}}{\mathrm{D} t}+\nabla \cdot \boldsymbol{u}_{\mathrm{f}}\right),
$$

describing how the liquid's local volume changes over time. The local volume change also causes a change in liquid pressure, which we will derive in $§ 3.4 .1$ by leveraging (16).

Remark: Consistency with non-mixture liquid. The momentum and mass conservation laws derived from mixture theory are general enough to describe non-mixture fluids as well. For example, when there is no strand in the mixture (i.e., $\epsilon_{\mathrm{f}}=1$ everywhere), the first term on the right hand side of (16) vanishes, and Eq. (16) becomes the standard mass conservation equation for a single-phase material.

\subsection{Coupling Forces}

We now focus on the coupling forces that allow bulk liquid, discrete strands, and surface flows to interact each other. In particular, we present force models between bulk liquid and submerged strands (§3.4.1 and §3.4.2) and between bulk liquid and the strands' surface flows (§3.4.3). Note that the interaction force between strands and their surface flows has already been discussed, emerging as the inertial force in the last term of (9).

3.4.1 Pressure. First, we derive the pressure for a non-Newtonian mixture. An expression for pressure is needed for two reasons: i) pressure drives the bulk liquid's motion by contributing to its stress in the momentum equation (14), and ii) the pressure gradient produces forces on the submerged strands, contributing to the external force term in (9). 


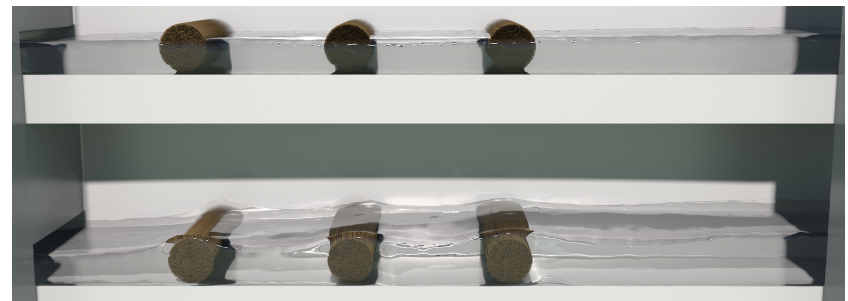

Fig. 7. Comparison between varying and fixed volume fraction (in the absence of drag). Liquid flows from left to right through strands fixed in place. Top: without the volume fraction considered. The liquid flow does not change its volume despite part of the space being occupied by strands; Bottom: with the volume fraction considered. The liquid will expand naturally as it passes through strands.

Pressure is caused by volume change of the liquid. Formally, as explained in supplemental $\S S 1$, it is the negated derivative of dilational potential energy $W_{v}$ with respect to elastic volume change $J^{E}$ (i.e., $\left.p \equiv-\frac{\partial W_{v}}{\partial J^{E}}\right)-$ akin to how a spring force is related to the spring's energy. There exist many models for the dilational potential energy, and our bulk liquid and strand models presented in earlier sections could incorporate any of them (through $p$ ). In our examples, we choose a modified neo-Hookean model [Simo 1988], following the work of Yue et al. [2015] (see supplemental §S1 for details).

For the sake of numerical stability our simulation uses an implicit integration scheme which requires, for its implicit pressure solve, the pressure's material derivative,

$$
\frac{\mathrm{D}_{\boldsymbol{u}_{\mathrm{f}}} p}{\mathrm{D} t}=-\frac{\partial^{2} W_{v}}{\partial J^{E^{2}}} \frac{\mathrm{D}_{\boldsymbol{u}_{\mathrm{f}}} J^{E}}{\mathrm{D} t} .
$$

Substituting (16) and the dilational potential energy (i.e., (S4) in supplemental §S1) into (17) reveals that the pressure's material derivative is related to both the liquid and strand velocities in the mixture:

$$
\begin{aligned}
\frac{\mathrm{D}_{\boldsymbol{u}_{\mathrm{f}}} p}{\mathrm{D} t} & =-\frac{\partial^{2} W_{v}}{\partial J^{E^{2}}} J^{E}\left(\epsilon_{\mathrm{f}}^{-1} \frac{\mathrm{D}_{\boldsymbol{u}_{\mathrm{f}}} \epsilon_{\mathrm{f}}}{\mathrm{D} t}+\nabla \cdot \boldsymbol{u}_{\mathrm{f}}-\frac{1}{J^{P}} \frac{\mathrm{D}_{\boldsymbol{u}_{\mathrm{f}}} J^{P}}{\mathrm{D} t}\right) \\
& =-\frac{\kappa}{2} J^{*}\left(\frac{1}{\epsilon_{\mathrm{f}} V^{*}} \sum_{i} V_{i} \nabla w_{R, i}(\boldsymbol{x}) \cdot\left(\boldsymbol{u}_{\mathrm{s}, i}-\boldsymbol{u}_{\mathrm{f}}\right)+\nabla \cdot \boldsymbol{u}_{\mathrm{f}}\right),
\end{aligned}
$$

where $J^{*}$ is a shorthand for $J^{*} \equiv\left(J^{E}+\frac{1}{J^{E}}\right)$. The second equality utilizes the material derivative of the liquid volume fraction, $\frac{\mathrm{D}_{\boldsymbol{u}_{\mathrm{f}}} \epsilon_{\mathrm{f}}}{\mathrm{D} t}$, estimated in (12) along with the fact that plastic flow is often isochoric (i.e., $J^{P}=1$ ) even under very high pressure [Bridgman 1949a,b]. Eq. (18b) will be discretized in $\S 4$ to solve for the pressure.

Pressure force on strands. When strands are mixed with the liquid, the spatial gradient of the pressure also induces a force $f_{p, i}$ on each submerged strand element $i$ through $f_{p, i}=V_{i} \nabla p\left(x_{i}\right)$, where $V_{i}$ is the volume of the discrete strand element $i$ centered at position $x_{i}$. The force $f_{p, i}$ contributes in part to the external force $f_{\text {ext,s }}$ in (9). As a result, we are able to correctly capture buoyancy effects (see Fig. 6 and the supplementary video).

Remark: Comparison to single-phase liquid. Our pressure equation differs from that used in previous work (e.g., [Stomakhin et al. 2014]) wherein only a single-phase liquid is considered. In particular, our

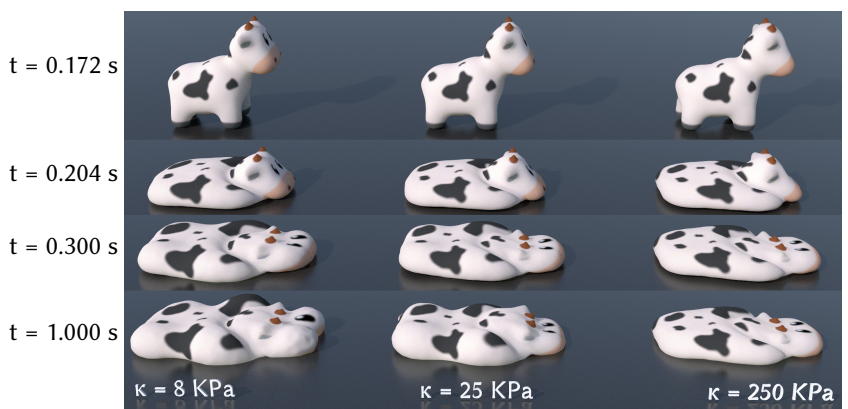

Fig. 8. Simulated cream with a wide range of bulk moduli $(\kappa)$. Cream with a lower bulk modulus $\kappa$ shrinks or dilates more easily during the simulation, and has a larger volume in the steady state.

model captures the mixture's effective volume change caused by the change of liquid volume fraction (as seen from the first term on the right hand side of (16)). This volume change in turn contributes to the pressure's material derivative (18a). In comparison to a single-phase liquid model, our mixture-theory-based model is able to capture richer liquid-strand interactions as illustrated in Fig. 7.

Remark: Comparison to incompressible mixture models. Prior work by Fei et al. [2018] and Gao et al. [2018a] developed models for incompressible mixtures. In supplemental $\S S 4$, we show that the incompressible mixture is a special case of our model when the material stiffness $\kappa$ (e.g., used in (18b)) approaches infinity. Therefore, our model is able to simulate liquids across a wider range of stiffnesses (see Fig. 8).

Remark: Generality. We obtain (18a) based only on compressible mixture theory; no specific properties of non-Newtonian fluids or the dilational potential model are needed. In other words, Eq. (18a) is sufficiently general to simulate other types of liquid materials (e.g., see [Bonet and Wood 1997; Smith et al. 2018]). In the same vein, the discrete solids mixed into the liquid in our model need not necessarily be strands; other types of solids, such as gravel, sand, and clay, could be readily simulated as well.

3.4.2 Drag Force. Next, we present a model for computing drag forces between the liquid and submerged strands. The drag force contributes to the external force terms for both strands (9) and the liquid (13)

We choose to employ a popular drag model proposed by Di Felice [1994]. This is a simple velocity-dependent model that is nevertheless flexible enough to support a range of materials and rheologies [Lagger et al. 2015; Li and Zhao 2018]. Concretely, the drag force of a discrete strand element $i$ centered at $\boldsymbol{x}_{\boldsymbol{i}}$ is expressed as

$$
f_{\text {drag, }, i}=\frac{1}{2} \rho_{\mathrm{f}}\left(\boldsymbol{x}_{i}\right) C_{\mathrm{d}} A_{\perp, i}\left\|\boldsymbol{u}_{\mathrm{f}}\left(\boldsymbol{x}_{i}\right)-\boldsymbol{u}_{\mathrm{s}, i}\right\|_{2} \epsilon_{\mathrm{f}}^{-\chi_{i}}\left(\boldsymbol{u}_{\mathrm{f}}\left(\boldsymbol{x}_{i}\right)-\boldsymbol{u}_{\mathrm{s}, i}\right),
$$

where $\boldsymbol{u}_{\mathrm{f}}$ and $\boldsymbol{u}_{\mathrm{s}, i}$ are liquid and strand element velocities (as defined in §3.3.1), $A_{\perp, i}$ is the area of the strand element $i$ projected on the plane perpendicular to the relative velocity vector $\boldsymbol{u}_{\mathrm{f}}-\boldsymbol{u}_{\mathrm{s}}, C_{\mathrm{d}}$ is the drag coefficient, and the parameter $\chi_{i}$ takes the empirical form

$$
\chi_{i}=3.7-0.65 \exp \left[-\frac{1}{2}\left(1.5-\log \operatorname{Re}_{\mathrm{p}, i}\right)^{2}\right],
$$




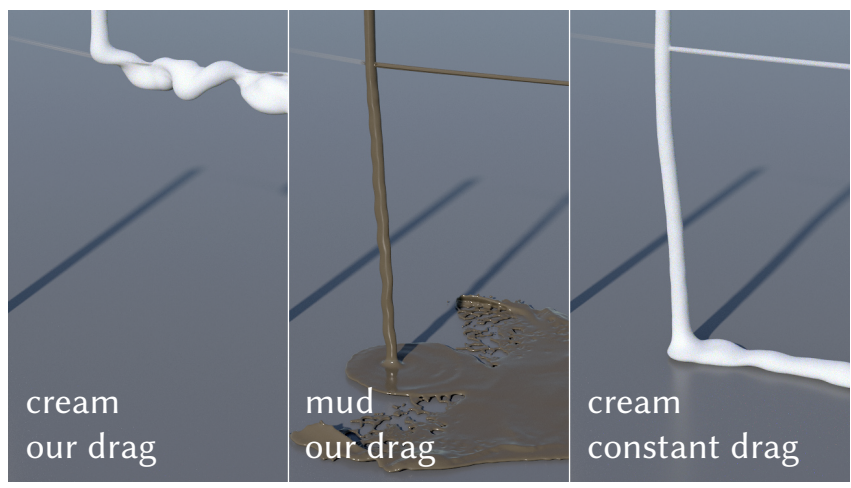

Fig. 9. Comparison of different drag coefficients. Streams of shaving cream and mud are poured onto seven hanging strands. Left and middle: Our drag model yields very different interaction behavior depending on the liquid type; Right: A constant drag coefficient, on the other hand, produces a drag effect for the shaving cream similar to that of mud, since the yield stress and viscosity are not considered.

in which $\mathrm{Re}_{\mathrm{p}, i}$ is $i$-th strand element's particle Reynolds number, whose specific formula is given in (S66) of the supplemental material.

A key parameter in this model is the drag coefficient $C_{\mathrm{d}}$. Experiments have shown that $C_{\mathrm{d}}$ can be taken as a constant value if the solid elements are spherical particles and the liquid has a low viscosity and a high Reynolds number [Zhong et al. 2016]. While recent work on simulating wet sand [Gao et al. 2018a] used this model with a constant $C_{\mathrm{d}}$, we found that a constant $C_{\mathrm{d}}$ in (19) limits the model's generalizability to different liquid materials, as illustrated in Fig. 9 and the supplementary video.

We adopt a formulation for $C_{\mathrm{d}}$ developed by Renaud [2004]. It has been extensively verified for predicting drag forces between irregularly shaped solid elements and liquids, both Newtonian and non-Newtonian, with a Reynolds number up to 1500 (see [Agarwal and Chhabra 2007; Chhabra 2006; Rajitha et al. 2006]). Details are provided in supplemental §S6, and its efficacy is demonstrated in Fig. 9.

We conclude by homogenizing the drag forces experienced by the discrete strand elements to apply the corresponding force on the liquid. Homogenization is performed by taking a kernel-weighted average of the drag on the discrete elements, similar to that in 3.3 .1 . That is,

$$
f_{\text {drag, },(\boldsymbol{x})}=\frac{1}{V^{*}} \sum_{i} w_{R, i}(\boldsymbol{x}) f_{\text {drag, }, i} .
$$

3.4.3 Constraints Between Bulk Liquid and Surface Flows. Lastly, we consider the interactions at the interface between the bulk liquid and the reduced surface flows. Mathematically, since the momentum conservation law is a second-order partial differential equation, it needs two boundary conditions on the interface to couple the surface flow with the bulk liquid.

Pressure boundary condition. The first condition requires that the pressure in the surface flow and the bulk liquid agree at the interface (see adjacent figure). As discussed in §3.1, the internal stress of the surface flow depends on the reduced shear strain $c_{\tau}^{\mathrm{E}}$. Thus, the surface flow pressure $p_{\tau}$ at the interface position $\boldsymbol{x}_{0}$ is $p_{\tau}\left(x_{0}\right)=-\mu c_{\tau}^{\mathrm{E}}\left(\boldsymbol{x}_{0}\right)$, where $\mu$ is the liquid's shear modulus. Let $p_{\mathrm{f}}\left(\boldsymbol{x}_{0}\right)$ be the bulk liquid pressure at the same interface position. Then, the Dirichlet pressure condition is

$$
p_{\mathrm{f}}\left(\boldsymbol{x}_{0}\right)=p_{\tau}\left(\boldsymbol{x}_{0}\right) .
$$

This boundary condition will be used in our numerical pressure solve in $\S 4$.

Velocity boundary condition. The second condition is similar to the liquid-solid boundary condition in a typical liquid simulation, demanding velocity agreement at the interface, namely,

$$
u_{\tau}\left(\boldsymbol{x}_{0}\right)=\boldsymbol{t}^{T}\left(\boldsymbol{u}_{\mathrm{f}}\left(\boldsymbol{x}_{0}\right)-\boldsymbol{u}_{\mathrm{s}, \mathrm{i}}\right),
$$

where the left hand side is the surface flow velocity, while the right hand side indicates the projected velocity difference (along strand direction $t$ ) between the bulk liquid and the strand element $i$ at $\boldsymbol{x}_{0}$.

We enforce this boundary condition by applying penalty forces on discrete strand

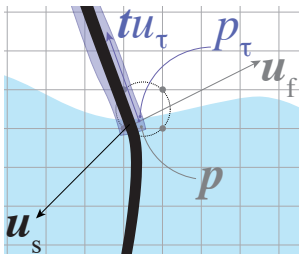
elements and the bulk liquid. This approach is similar to the classic immersed boundary method for simulating liquid-solid coupling [Peskin 2002]. The penalty force at a strand element $i$ is computed as

$$
f_{\mathrm{eq}, i}=-\frac{1}{h} m_{\tau, i}\left(\boldsymbol{t}_{i} u_{\tau, i}+\boldsymbol{u}_{\mathrm{s}, i}-\boldsymbol{u}_{\mathrm{f}}\right),
$$

where $h$ is the timestep size, $m_{\tau, i}$ is the mass of liquid on strand element $i$, and $u_{\tau, i}$ is the flow velocity at the strand element $i$. In the strand's momentum equation, (9), $f_{\text {eq, } i}$ serves as a part of the external force $f_{\text {ext,s. }}$. The corresponding penalty force on the liquid is the homogenization of the discrete element forces $f_{\text {eq, } i}$ (i.e., $\left.f_{\text {eq,f }}(\boldsymbol{x})=-\sum_{i} w_{R, i}(\boldsymbol{x}) f_{\mathrm{eq}, i}\right)$. The force $f_{\mathrm{eq}, \mathrm{f}}$ contributes to the external force $f_{\text {ext,f }}$ in the liquid's momentum equation, (13).

\subsection{Contact Between Wet Strands}

Inter-strand contacts also significantly affect strand motion. In the context of wet strands, a straightforward approach to resolve contacts is through penalty forces. The penalty force can produce both repulsive and cohesive effects [Fei et al. 2017; Lin 2014, 2015], because of the liquid bridge that forms between two strands. However, penalty force methods suffer from several critical disadvantages: Wet strands, such as wet hair, are known to exhibit a strong frictional effect [Bhushan et al. 2005], but it remains unclear how to incorporate a principled friction model into penalty methods. Moreover, penalty forces on strands can become strongly cohesive due to the liquid bridge in between, quickly causing strand penetration during the simulation (see Fig. 10). The nature of non-Newtonian liquids makes matters worse, as such liquids may produce even stronger strand cohesion due to non-Newtonian elasticity.

In light of these factors, we instead seek to resolve contacts through a constraint-based method that solves a second-order Coulomb cone (SOCC) problem for both contact and friction forces, following prior work [Bertails-Descoubes et al. 2011; Daviet et al. 2011; Jean 1999; Moreau 1988]. This approach ensures contact resolution without strand penetration and ensures that the friction forces follow Coulomb's law of friction precisely. However, it traditionally assumes that the contact force can be repulsive, but not cohesive, which is not an appropriate assumption for our setting. 


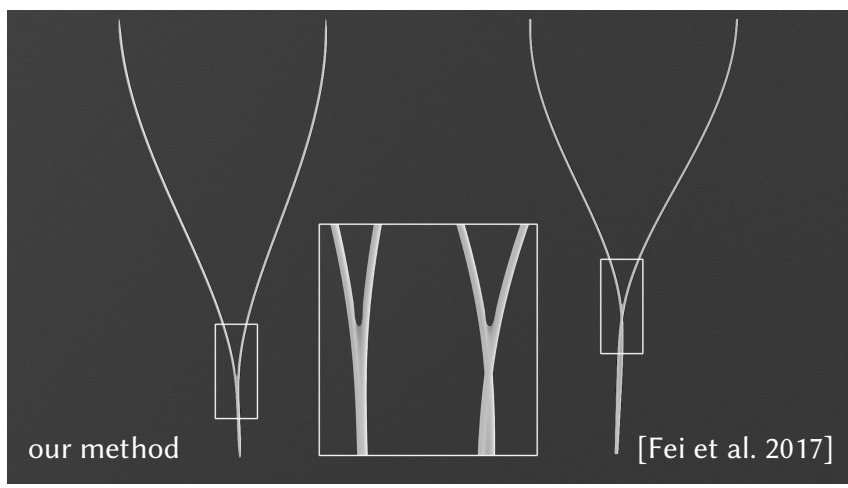

Fig. 10. Comparison between constraint- and penalty-based collision. Even with relatively large cohesion forces (cream), our method (Left) can correctly handle contact with cohesion, without the tunneling problem seen in the penalty method (Right).

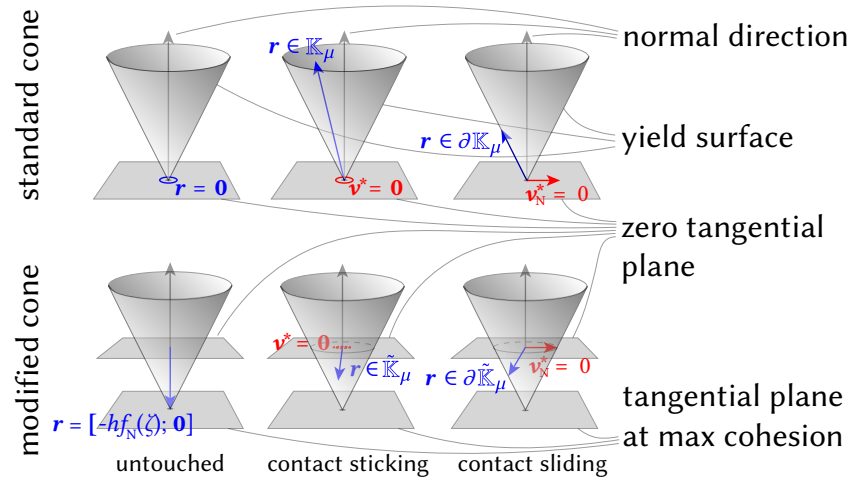

Fig. 11. Top: Regular second-order Coulomb cone (SOCC) when the strands are dry. An impulse $r$ is constrained by the cone when two rods collide. For the tangential part of $\boldsymbol{r}$ (denoted $r_{\mathrm{T}}$ ) we have either $\boldsymbol{v}^{*}=0$ when $r_{\mathrm{T}} \in K_{\mu}$, producing static friction (sticking), or $\left\|\boldsymbol{v}_{\mathrm{T}}^{*}\right\|>0$ when $r_{\mathrm{T}}$ reaches the yield surface, i.e., $r_{\mathrm{T}} \in \partial K_{\mu}$, producing dynamic friction (sliding). The relative velocity in the normal direction will be eliminated for either the sticking or sliding case. Bottom: Modified SOCC applied when the strands are covered with liquid. The impulse $\boldsymbol{r}$ is offset and may yield cohesion: as long as the cohesive impulse is less than $h f_{\mathrm{N}}$, i.e., $r_{\mathrm{N}}>-h f_{\mathrm{N}}$, we have $v_{\mathrm{N}}^{*}=0$. Due to the offset of the cone, an additional frictional effect will be induced, which, physically, corresponds to the shear stress of the cohesive material. Finally, when $f_{N}=0$, the modified SOCC degenerates to a standard one.

To address this limitation, we propose an augmented Coulomb cone model.

3.5.1 A modified second-order cone. We first introduce some notation for the regular Coulomb cone [Bertails-Descoubes et al. 2011; Daviet et al. 2011]. Given a contact point where the surface normal is denoted by $\boldsymbol{n}$, we consider the contact impulse (rather than force) across a timestep size $h$. The contact impulse, including both the normal and frictional impulses, is denoted by $r$. We use the subscripts $\mathrm{N}$ and $\mathrm{T}$ to denote the vector components along the normal direction and on the tangential plane, respectively. For example, $r_{\mathrm{N}}=\boldsymbol{n}^{T} \boldsymbol{r}$ is the normal impulse (i.e., a scalar) and $\boldsymbol{r}_{\mathrm{T}}=\left(\mathbf{I}_{3}-\boldsymbol{n} \boldsymbol{n}^{T}\right) \boldsymbol{r}$ is the tangential (frictional) impulse (i.e., a vector). We also express $\boldsymbol{r}$ explicitly using its two components as $\left[r_{\mathrm{N}} ; \boldsymbol{r}_{\mathrm{T}}\right]$.
With this notation, the regular SOCC $\mathbb{K}_{\mu}$, defined under a friction coefficient $\mu$, is the set of vectors containing all possible contact impulses satisfying Coulomb's law of friction,

$$
\mathbb{K}_{\mu}=\left\{\left[r_{\mathrm{N}} ; \boldsymbol{r}_{\mathrm{T}}\right] \mid \mu x_{\mathrm{N}} \geq\left\|\boldsymbol{x}_{\mathrm{T}}\right\|_{2}\right\},
$$

which is visualized in the top row of Fig. 11.

Next, we extend this SOCC to incorporate cohesive forces between wet strands. When a liquid bridge connects two wet strands, a cohesive force arises due to the liquid's surface tension and elasticity. Denoted as $f_{\mathrm{N}}(\zeta)$, this force depends on the strand distance $\zeta$ and points along the normal direction $\boldsymbol{n}$, and its details will be discussed shortly. Now the total impulse $\boldsymbol{r}_{\mathrm{s}}$ includes both the contact impulse $r$ and the cohesive impulse $h f_{\mathrm{N}}(\zeta)$, so

$$
\boldsymbol{r}_{\mathrm{s}}=\boldsymbol{r}+h\left[f_{\mathrm{N}}(\zeta) ; \mathbf{0}\right] .
$$

The normal and tangential components of the total impulse $\boldsymbol{r}_{\mathrm{S}}$ must satisfy Coulomb's law of friction, that is, $\boldsymbol{r}_{\mathrm{s}}$ must reside in $\mathbb{K}_{\mu}$. This means that all possible contact impulses $\boldsymbol{r}$ form a different SOCC (denoted as $\tilde{\mathbb{K}}_{\mu}$ ), that is like $\mathbb{K}_{\mu}$ but translated along the normal direction by $-h\left[f_{\mathrm{N}}(\zeta) ; 0\right]$. The bottom row of Fig. 11 shows a visualization of $\tilde{\mathbb{K}}_{\mu}$.

Given $\tilde{\mathbb{K}}_{\mu}$, there are several outcomes for the relative velocity $\boldsymbol{v}^{*}$ of two strand elements after their contact is resolved, depending on where the contact impulse locates in the modified SOCC:

(a) Untouched: $\zeta>\zeta_{0}, r_{\mathrm{s}}=0$, and $\boldsymbol{v}^{*}$ is not affected,

(b) Contact sticking: $\zeta=\zeta_{0}, \boldsymbol{r}_{\mathrm{s}} \in \mathbb{K}_{\mu}, \boldsymbol{r} \in \tilde{\mathbb{K}}_{\mu}$, and $\boldsymbol{v}^{*}=0$,

(c) Contact sliding: $\zeta=\zeta_{0}, r_{\mathrm{s}} \in \partial \mathbb{K}_{\mu}, \boldsymbol{r} \in \partial \tilde{\mathbb{K}}_{\mu}, v_{\mathrm{N}}^{*}=0$, and $\exists \alpha \in \mathbb{R}_{+}, \boldsymbol{v}_{\mathrm{T}}^{*}=-\alpha \boldsymbol{r}_{\mathrm{s}, \mathrm{T}}$.

Here $\partial \mathbb{K}_{\mu}$ denotes the boundary of the SOCC $\mathbb{K}_{\mu}$, and $\zeta_{0}$ is a critical distance between two strand elements, indicating when the impulse $\boldsymbol{r}_{s}$ occurs. The specific value of $\zeta_{0}$ deserves some careful reasoning, as will be discussed next.

3.5.2 Contact with hysteresis. In our augmented SOCC model, two strand elements stop moving towards one another when their distance reaches $\zeta_{0}$. In reality, two strands can approach until their surfaces touch each other, regardless of their surface flow thickness. This observation suggests that $\zeta_{0}$ should be set as $2 r$ where $r$ is the strand radius. On the other hand, when two strand elements move apart, the cohesive force persists until the liquid bridge between them breaks. This observation, by contrast, suggests that $\zeta_{0}$ should be a value related to the surface flow thickness.

We resolve this paradox by introducing contact hysteresis, in which the critical distance $\zeta_{0}$ for approaching strands is different from that of separating strands:

(a) Approaching: when $v_{\mathrm{N}}<0, \zeta_{0}=2 r$,

(b) Separating: when $v_{\mathrm{N}} \geq 0, \zeta_{0}=2 r+(1+0.5 \theta) \sqrt{A_{L}}$, where $A_{L}$ is the total area of the cross section of the liquid bridge, and $\theta$ is the contact angle determined by the liquid and strand materials.

In case (b), $\zeta_{0}$ is set to be the distance at which the liquid bridge breaks. To estimate this distance value, we follow the formula by Lian et al. [1993]. The hysteretic contact force profile is illustrated in Fig. 12. 

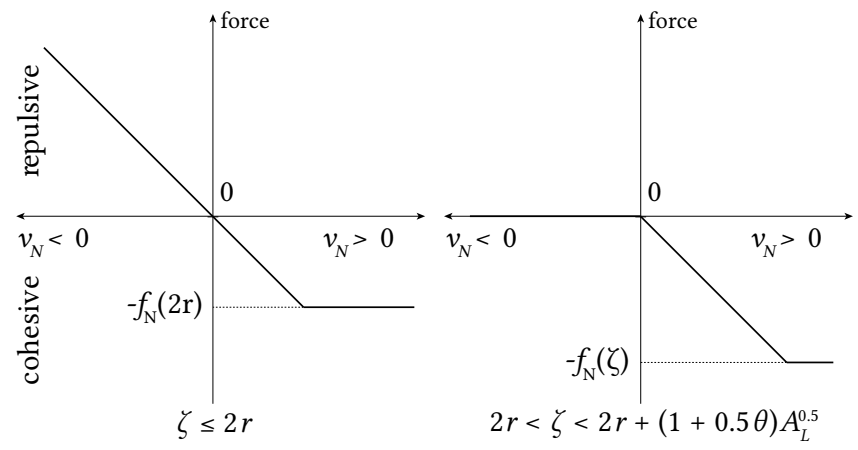

Fig. 12. The profile of the contact-cohesion force in the normal direction. Left: the solid parts of the strands touch each other. When the two strands tend to approach each other with negative unconstrained normal velocity, there will be a repulsive force; when the two strands tend to depart from each other with positive unconstrained normal velocity, there will be a cohesive force. We evaluate the cohesive force $f_{\mathrm{N}}$ at $2 r$ for the case $\zeta<2 r$ to avoid singularity. Right: the solid parts of the strands do not touch, but the liquid bridge exists. The two strands can freely move toward each other with negative unconstrained normal velocity, but a cohesive force will stick them together as long as they tend to separate with positive unconstrained normal velocity. In both cases, whenever there is a cohesive force, the relative normal velocity after applying constraint will be zero unless the maximal cohesive force is reached.

Solver. Similar to the standard SOCC problem, our augmented SOCC problem can be reformulated as a root-finding problem and solved by an existing solver [Bertails-Descoubes et al. 2011; Daviet et al. 2011]. We refer the reader to supplemental §S7 for details.

Remark. When two strands approach (i.e., $v_{\mathrm{N}}<0$ ), our contact hysteresis does not induce cohesive forces even if the liquid bridge has formed, unless the strand surfaces touch each other. However, the cohesive forces appear when they move away from each other. While not entirely accurate, our model nevertheless captures interesting wet strand behaviors, such as the formation of strand bundles. This is because when (sufficiently close) strands attempt to move apart, the cohesive force tends to prevent them from separating. The lack of cohesion as strands approach has an advantage in practice: the cohesion force does not act to accelerate the negative normal velocity and thereby increase the speed of the collision. Otherwise, the cohesive force would render the system much stiffer, making strand penetration or tunneling much harder to avoid.

3.5.3 Cohesive force. Cohesion is usually caused by the capillary surface energy of the liquid, be it Newtonian or non-Newtonian. In addition, the non-Newtonian liquid may introduce an extra cohesive force: when two strands move apart and the liquid bridge gets stretched, the non-Newtonian liquid will experience a viscoplastic deformation until the bridge breaks. During a stretching motion, the elastic stress effectively produces an extra cohesive force on the strands. On the other hand, the non-Newtonian liquid bridge possesses a maximal stress, after which point the liquid begins to yield and convert its elastic strain into plastic strain. Thus the elastic stress is limited by the maximal stress.

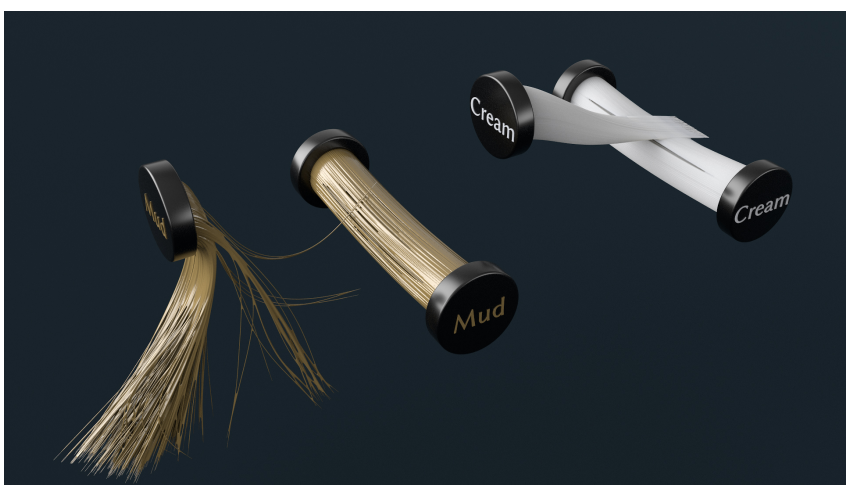

Fig. 13. Contact between strands with surface flows of different materials-Left: mud; Right: milk cream.

Therefore, we model the cohesive force $f_{\mathrm{N}}$ with two components: the capillary part $f_{\mathrm{N}, \mathrm{c}}$ and viscoplastic part $f_{\mathrm{N}, \mathrm{v}}$, that is,

$$
f_{\mathrm{N}}=f_{\mathrm{N}, \mathrm{v}}+f_{\mathrm{N}, \mathrm{c}}
$$

For the capillary part $f_{\mathrm{N}, \mathrm{c}}$, we adopt the cohesive force profile proposed by Fei et al. [2017], whose detailed formulation is described in supplemental §S8 for completeness.

The viscoplastic part $f_{\mathrm{N}, \mathrm{v}}$ can be estimated from the flow rate function (see (S13) in supplemental §S1). In particular, following Atanackovic and Guran [2012], we compute $f_{\mathrm{N}, \mathrm{v}}$ by estimating the stress applied on the cross sectional area $A_{c}$ of the liquid bridge:

$$
f_{\mathrm{N}, \mathrm{v}}=A_{c}\left[\sqrt{\frac{2}{3}} \tau_{Y}+\eta \gamma^{n}\right],
$$

where $\tau_{Y}$ is the yield stress, $\eta$ is the flow consistency index, and $n$ is the flow behavior index.

To use (28) in practice, we estimate the flow rate $\gamma=\frac{\partial\left(\boldsymbol{n}^{T} \boldsymbol{u}_{s}\right)}{\partial \boldsymbol{n}}$ using finite differences, and $A_{c}$ using the area where the rod $A_{c}$ bridge liquid contacts the strand, i.e., $A_{c}=2 r \alpha \mathrm{d} l$ where $\mathrm{d} l$ is the wet length and $\alpha$ is the angle between the direction toward the liquid bridge and the direction toward the liquid/solid boundary.

\section{DISCRETIZATION}

With our continuous physical models in hand, we can proceed to discretization. We adopt four types of Lagrangian variables to discretize the simulated geometry: 1) traditional MPM particles, or particles for brevity, are used to discretize the bulk liquid; 2) Lagrangian rod vertices, or vertices, are used to discretize the elastic rods; 3) Lagrangian rod elements (segments), or elements, are used to set up a staggered discretization of the surface flow on the rods. In addition, the bulk liquid is solved on an Eulerian staggered grid with velocities stored on cell faces and pressure stored on cell centers, similar to the augmented material point method (AMPM) [Stomakhin et al. 2014]. Because our discretization shares similarities with other approaches for MPM [Daviet and Bertails-Descoubes 2017; Fei et al. 2018; Gao et al. 2018a; Jiang et al. 2017, 2016; Stomakhin et al. 2014; Tampubolon et al. 2017] and discrete elastic rods [Bergou et al. 2010, 2008; Kaufman et al. 2014], in the following we briefly cover 
Table 1. Notation for Discrete Variables.

\begin{tabular}{|c|c|c|}
\hline Symbol & Physical quantity & Dimension \\
\hline$q_{\mathrm{s}}$ & strand vertex position $(\mathrm{cm})$ & $3 n \times 1$ \\
\hline$q_{\mathrm{f}}$ & liquid particle position $(\mathrm{cm})$ & $3 p \times 1$ \\
\hline$\zeta$ & gaps between strands in contact $(\mathrm{cm})$ & $k \times 1$ \\
\hline$x_{i}$ & $i$-th face position $(\mathrm{cm})$ & $3 \times 1$ \\
\hline$\hat{\boldsymbol{x}}_{i}$ & predicted $i$-th face position $(\mathrm{cm})$ & $3 \times 1$ \\
\hline $\boldsymbol{e}_{i}$ & $i$-th face direction & $3 \times 1$ \\
\hline $\boldsymbol{u}_{\mathrm{s}}$ & strand vertex velocity $(\mathrm{cm} / \mathrm{s})$ & $3 n \times 1$ \\
\hline$u_{\mathrm{f}}$ & liquid particle velocity $(\mathrm{cm} / \mathrm{s})$ & $3 p \times 1$ \\
\hline$v$ & strand velocity in contact space $(\mathrm{cm} / \mathrm{s})$ & $3 k \times 1$ \\
\hline$r$ & contact impulse (dyn $\cdot s)$ & $3 k \times 1$ \\
\hline$p$ & liquid pressure $\left(\right.$ dyn $\left.\cdot \mathrm{cm}^{2}\right)$ & $c \times 1$ \\
\hline $\mathrm{G}_{\mathrm{w}, \mathrm{cn}}$ & gradient on vertices (from centers, $\mathrm{cm}^{-1}$ ) & $3 n \times c$ \\
\hline $\mathrm{G}_{\mathrm{w}, \mathrm{cn}}^{\mathrm{T}}$ & (negative) divergence on centers (from vertices, $\mathrm{cm}^{-1}$ ) & $c \times 3 n$ \\
\hline $\mathrm{G}_{\mathrm{cf}}$ & gradient on faces (from centers, $\mathrm{cm}^{-1}$ ) & $f \times c$ \\
\hline $\mathrm{G}_{\mathrm{cf}}^{\mathrm{T}}$ & (negative) divergence on centers (from faces, $\mathrm{cm}^{-1}$ ) & $c \times f$ \\
\hline $\mathbf{W}_{\mathrm{fn}}$ & weight mapping faces to vertices & $3 n \times f$ \\
\hline $\mathbf{W}_{\mathrm{fn}}^{\mathrm{T}}$ & weight mapping vertices to faces & $f \times 3 n$ \\
\hline $\mathrm{E}^{\mathrm{In}}$ & mapping contact space to world space & $3 n \times 3 k$ \\
\hline $\mathbf{W}_{\mathrm{fe}}$ & weight mapping faces to elements & $3 e \times f$ \\
\hline $\mathbf{W}_{\mathrm{fe}}^{\mathrm{T}}$ & weight mapping elements to faces & $f \times 3 e$ \\
\hline$W_{\text {ne }}$ & weight mapping vertices to elements & $e \times n$ \\
\hline $\mathrm{W}_{n e}^{\mathrm{T}}$ & weight mapping elements to vertices & $n \times e$ \\
\hline$T$ & mapping element tangent space to world space & $3 e \times e$ \\
\hline $\mathbf{H}_{\mathrm{s}}$ & Jacobian of the strand force $(\mathrm{dyn} / \mathrm{cm})$ & $3 n \times 3 n$ \\
\hline $\mathrm{H}_{\mathrm{f}}$ & Jacobian of the liquid's shear force $(\mathrm{dyn} / \mathrm{cm})$ & $f \times f$ \\
\hline $\mathbf{M}_{\mathrm{s}}$ & mass matrix of the strands $(\mathrm{g})$ & $3 n \times 3 n$ \\
\hline $\mathbf{M}_{\mathrm{f}}$ & mass matrix of the liquid (g) & $f \times f$ \\
\hline$V_{f}$ & volume of liquid at faces $\left(\mathrm{cm}^{3}\right)$ & $f \times f$ \\
\hline $\mathrm{V}_{\mathrm{c}}$ & volume of liquid at centers $\left(\mathrm{cm}^{3}\right)$ & $c \times c$ \\
\hline $\mathrm{V}_{\mathrm{s}}$ & volume of vertices $\left(\mathrm{cm}^{3}\right)$ & $3 n \times 3 n$ \\
\hline $\mathrm{V}_{\mathrm{p}}$ & volume of particles $\left(\mathrm{cm}^{3}\right)$ & $3 p \times 3 p$ \\
\hline $\mathbf{M}_{\tau, \mathrm{n}}$ & mass matrix of flow on vertices $(\mathrm{g})$ & $n \times n$ \\
\hline$A_{\tau}$ & surface flow area $\left(\mathrm{cm}^{2}\right)$ & $n \times 1$ \\
\hline $\boldsymbol{u}_{\tau}$ & surface flow velocity $(\mathrm{cm} / \mathrm{s})$ & $e \times 1$ \\
\hline
\end{tabular}

the common aspects while emphasizing the novel aspects of our approach in detail.

Notation. Table 1 defines our variable notation for a scenario containing $p$ particles, $n$ vertices, $e$ elements, $k$ contacts, $f$ faces, and $c$ centers. We adopt the superscript $t$ to indicate variables that are known at the beginning of the current time step, $i$ to indicate variables in the $i$-th Newton iteration, and $t+1$ to indicate variables to be solved for at the end of the time step. The time step size is denoted as $h$.

\subsection{Discrete Constrained Dynamics}

A naive but accurate approach would be to solve the strand, the bulk liquid, and the surface flow simultaneously. Unfortunately, since the velocities of these three components are tightly coupled into a stiff, non-smooth, and non-symmetric system, it can be very difficult to solve in practice, especially as sufficient nonlinearity for collisions is usually required for stability of the strands [Kaufman et al. 2014].

Therefore, in this work we adopt a staggered integrator and update the variables of different phases in an alternating fashion. One resulting benefit is greater ease of implementation: we can adopt existing methods for strand simulation (e.g., we adopt the method of Kaufman et al. [2014]) and non-Newtonian liquids (e.g., we adopt the Herschel-Bulkley liquid model of Yue et al. [2015]), and simply enhance them to support the coupling between the strands and the non-Newtonian liquid.

Strand Simulation. Each time step begins with surface flow and strand simulation. We first apply semi-Lagrangian advection to the mass and velocity of the surface flow on the strand [Stam 1999]. Then we integrate the strand dynamics, temporarily assuming the bulk liquid pressure is zero. The solved strand velocity is used for collision detection and as a prediction for the later pressure solve and additional surface flow dynamics. Discretizing (9) leads to the discrete strand dynamics equation,

$$
\begin{aligned}
\left(\mathbf{M}_{\mathrm{s}}^{*}+h \mathbf{D}_{\mathrm{s}}\right) \boldsymbol{u}_{\mathrm{s}}^{t+1} & =\mathbf{M}_{\mathrm{s}}^{*} \boldsymbol{u}_{\mathrm{s}}^{t}+h\left(f_{\mathrm{int}}^{t+1}+\mathbf{M}_{\mathrm{s}} \boldsymbol{g}+\mathbf{D}_{\mathrm{s}} \mathbf{W}_{\mathrm{fn}} \boldsymbol{u}_{\mathrm{f}}^{t}\right) \\
& +\mathbf{M}_{\tau, n}^{*}\left(\boldsymbol{u}_{\mathrm{s}}^{t}-\boldsymbol{u}_{\mathrm{s},-\boldsymbol{u}_{\tau, n}^{t}}^{t}\right),
\end{aligned}
$$

where $\mathbf{M}_{\mathrm{S}}^{*}$ is the combined mass of the strand and the surface flow on it (after it has been advected for the current time step). The term $\mathbf{M}_{\tau, n}^{*}\left(\boldsymbol{u}_{\mathrm{s}}^{t}-\boldsymbol{u}_{\mathrm{s},-\boldsymbol{u}_{\tau, n}^{t}}^{t}\right)$ is the extra rod inertia induced by the surface flow, which is also computed with semi-Lagrangian advection [Stam 1999]: the backtraced velocity of the $k$-th vertex is defined as

$$
\boldsymbol{u}_{\mathrm{s},-\boldsymbol{u}_{\tau, \mathrm{n}}^{t}, k}^{t} \equiv \boldsymbol{u}_{\mathrm{s}}\left(q_{\mathrm{s}, \tau}-h u_{\tau, \mathrm{n}}^{t}\right)_{\text {interp }}
$$

where $q_{s, \tau}$ is the strand-space coordinate of the vertex, and the function $\boldsymbol{u}_{s}(x)_{\text {interp }}$ interpolates the value from $\boldsymbol{u}_{s}$ at strand coordinate $x$.

To solve the discrete strand equation, we adopt a nonlinear NewtonRaphson solver [Kaufman et al. 2014]. For the $i$-th Newton iteration, the linearized equation is

$$
\begin{aligned}
& \mathrm{C}_{\mathrm{s}} \boldsymbol{u}_{\mathrm{s}}^{i+1}=\alpha^{i}\left(\mathbf{M}_{\mathrm{s}}^{*}\left(\boldsymbol{u}_{\mathrm{s}}^{t}-\boldsymbol{u}_{\mathrm{s}}^{i}\right)+h\left(f_{\text {int }}^{t}+\mathbf{M}_{\mathrm{s}}^{*} \boldsymbol{g}\right)\right. \\
& \left.+h \mathrm{D}_{\mathrm{s}} \mathbf{W}_{\mathrm{fn}} \boldsymbol{u}_{\mathrm{f}}^{t}+\mathbf{M}_{\tau, n}^{*}\left(\boldsymbol{u}_{\mathrm{s}}^{t}-\boldsymbol{u}_{\mathrm{s},-\boldsymbol{u}_{\tau, n}^{t}}^{t}\right)\right)+\mathrm{C}_{\mathrm{s}} \boldsymbol{u}_{\mathrm{s}}^{i}
\end{aligned}
$$

where $\alpha$ is the step length computed with backtracking line search [Armijo 1966], $\mathbf{C}_{\mathrm{s}}=\mathbf{M}_{\mathrm{S}}^{*}+h \mathbf{D}_{\mathrm{s}}+h^{2} \mathbf{H}_{\mathrm{s}}$ is the the combination of mass and force Jacobian matrix, and $\boldsymbol{u}_{\tau, \mathrm{n}}^{t} \equiv \mathbf{W}_{\mathrm{ne}}^{\mathrm{T}} \boldsymbol{u}_{\tau}^{t}$ is the surface flow velocity mapped to vertices. A complex step of DER is computing the gradient and Hessian of the discrete curvatures (which are then used to compute $f_{\text {int }}^{t}$ and $\mathbf{H}_{\mathrm{s}}$ ). To assist potential readers to reproduce, we present a detailed derivation in supplemental §S10.

After the pressure and surface flow have been solved, we reintegrate the dynamics of the strand, this time using the solved pressure gradient and updated surface flow mass (hence the updated combined mass is denoted as $\mathbf{M}^{t+1}$ below), where the $i$-th iteration of the momentum equation's Newton solve becomes

$$
\begin{array}{r}
\mathbf{C}_{\mathrm{s}} \boldsymbol{u}_{\mathrm{s}}^{i+1}=\alpha^{i}\left(\mathbf{M}_{\mathrm{s}}^{t+1}\left(\boldsymbol{u}_{\mathrm{s}}^{t}-\boldsymbol{u}_{\mathrm{s}}^{i}\right)+h\left(f_{\text {int }}^{t}+\mathbf{M}_{\mathrm{s}}^{t+1} \boldsymbol{g}\right)\right. \\
\left.+h \mathbf{D}_{\mathrm{s}} \mathbf{W}_{\mathrm{fn}} \boldsymbol{u}_{\mathrm{f}}^{t}+\mathbf{M}_{\tau, n}^{t+1}\left(\boldsymbol{u}_{\mathrm{s}}^{*}-\boldsymbol{u}_{\mathrm{s},-\boldsymbol{u}_{\tau, n}^{*}}^{t+1}\right)-h \mathbf{V}_{\mathrm{s}} \mathbf{G}_{\mathrm{w}, \mathrm{cn}} \boldsymbol{p}\right)+\mathbf{C}_{\mathrm{s}} \boldsymbol{u}_{\mathrm{s}}^{i} .
\end{array}
$$

Since the surface flow velocity has been updated at this point, we compute the backtraced velocity with the updated surface flow velocity $\boldsymbol{u}_{\tau, n}^{t+1}$ and the (unconstrained) predicted solid velocity $\boldsymbol{u}_{\mathrm{s}}^{*}$. We then use the solved velocity (denoted by $\boldsymbol{u}_{\mathrm{s}}^{\dagger}$ below) for contact resolution. 


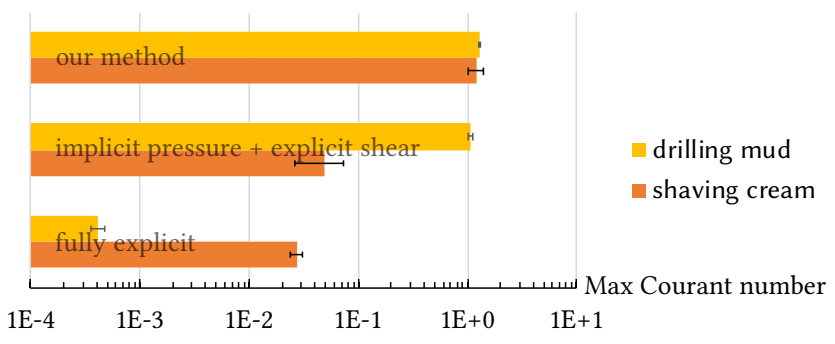

Fig. 14. Comparing implicit and explicit integration. We compare the maximal Courant number allowed for stable simulation between different integrators for the scene in Figure 9. The error bars indicate standard deviation over different grid settings. We adopt logarithmic coordinates and put the origin at $10^{-4}$ for legibility.

Finally, after the contact impulse $\boldsymbol{r}$ is solved, we update the velocity with the impulse added to the right hand side, using

$$
\begin{array}{r}
\mathrm{C}_{\mathrm{s}} \boldsymbol{u}_{\mathrm{s}}^{i+1}=\alpha^{i}\left(\mathbf{M}_{\mathrm{s}}\left(\boldsymbol{u}_{\mathrm{s}}^{t}-\boldsymbol{u}_{\mathrm{s}}^{i}\right)+h\left(f_{\mathrm{int}}^{t}+\mathbf{M}_{\mathrm{s}} \boldsymbol{g}\right)\right. \\
\left.+h \mathbf{D}_{\mathrm{s}} \mathbf{W}_{\mathrm{fn}} \boldsymbol{u}_{\mathrm{f}}^{t}+\mathbf{M}_{\tau, n}\left(\boldsymbol{u}_{\mathrm{s}}^{\dagger}-\boldsymbol{u}_{\mathrm{s},-\boldsymbol{u}_{\tau, n}^{t+1}}^{\dagger}\right)-h \mathbf{V}_{\mathrm{s}} \mathbf{G}_{\mathrm{w}, \mathrm{cn}} \boldsymbol{p}+\mathbf{E} \boldsymbol{r}\right)+\mathrm{C}_{\mathrm{s}} \boldsymbol{u}_{\mathrm{s}}^{i}
\end{array}
$$

Semi-Implicit Herschel-Bulkley Liquid. Explicitly integrated HerschelBulkley liquid is only stable with a time step that is two orders of magnitude smaller [Yue et al. 2015] than the implicitly integrated strands. To match the time step of the strands, the pressure and the shear stress must be implicitly integrated. Similar to prior work [Stomakhin et al. 2014], we adopt a splitting scheme when integrating the shear stress and pressure for computational efficiency, which leads to a stable semi-implicit integrator. Figure 14 compares our method against explicit integration; our semi-implicit method can stably handle much higher Courant numbers.

We first integrate the shear stress without the pressure applied by solving

$$
\mathrm{C}_{\mathrm{f}} \boldsymbol{u}_{\mathrm{f}}^{*}=\mathbf{M}_{\mathrm{f}} \boldsymbol{u}_{\mathrm{f}}^{t}+h\left(f_{\mathrm{MPM}}^{t}+\mathbf{M}_{\mathrm{f}} \boldsymbol{g}+\mathrm{D}_{\mathrm{f}} \boldsymbol{u}_{\mathrm{s}}^{*}\right)
$$

where $\mathbf{C}_{\mathrm{f}}=\mathbf{M}_{\mathrm{f}}+\mathbf{D}_{\tau}+h \mathbf{D}_{\mathrm{f}}+h^{2} \mathbf{H}_{\mathrm{f}}^{t}, \mathbf{D}_{\mathrm{f}}=\operatorname{diag}\left(\mathbf{W}_{\mathrm{fn}}^{\mathrm{T}} \operatorname{vec}(\mathbf{D})\right) \in \mathbb{R}^{f \times f}$ is a diagonal matrix containing all drag coefficients interpolated on the grid, and $\mathbf{H}_{\mathrm{f}}^{t}$ is the Jacobian matrix of shear force evaluated at time step $t$ (see supplemental $\S S 11$ for its detailed computation). The operator vec(.) converts a diagonal matrix into a vector and $\operatorname{diag}(\cdot)$ converts a vector into a diagonal matrix. Similarly $\mathbf{D}_{\tau}=\operatorname{diag}\left(\mathbf{W}_{\mathrm{fn}}^{\mathrm{T}} \operatorname{vec}\left(\mathbf{M}_{\tau}\right)\right)$ is a diagonal matrix containing all the mass of the surface flow interpolated onto the grid. Here $f_{\text {MPM }}$ is the MLS-MPM discretization of the shear force [Hu et al. 2018] whose term on face $i$ can be computed as

$$
f_{\mathrm{MPM}, \mathrm{i}}^{t}=-\sum_{p} \mu J_{p}^{-2 / 3} N_{i}\left(\boldsymbol{x}_{p}\right) V_{p}^{0} \mathbf{D}_{p}^{-1} \boldsymbol{e}_{i}^{\mathrm{T}} \operatorname{dev}\left(\mathbf{b}_{p}^{E, t}\right)\left(\boldsymbol{x}_{i}-\boldsymbol{x}_{p}\right)
$$

where $\mu$ is the shear modulus; $N_{i}\left(x_{p}\right)$ is the B-spline kernel evaluated at the position $\boldsymbol{x}_{p}$ of particle $p ; \boldsymbol{x}_{f, i}$ is the central position of grid face $i ; \boldsymbol{e}_{i}$ is the normal direction of grid face $i ; \mathbf{D}_{p}$ is the inertia tensor of the kernel function; and $\operatorname{dev}\left(\mathbf{b}_{p}^{E, t}\right)$ is the deviatoric part of the left Cauchy-Green strain tensor $\mathbf{b}_{p}^{E, t}$ (refer to supplemental §S1 for a background introduction). We adopt a quadratic kernel where $\mathbf{D}_{p}=\frac{1}{4} \Delta x^{2} \mathbf{I}_{3}$ [Jiang et al. 2016] and $\Delta x$ is the grid spacing.

Using the new velocity with the shear stress applied, we solve the pressure equation (refer to Pressure Solve below for details). We then apply the pressure gradient onto the right hand side of (36) and perform another implicit solve, where

$$
\mathrm{C}_{\mathrm{f}} \boldsymbol{u}_{\mathrm{f}}^{t+1}=\mathbf{M}_{\mathrm{f}} \boldsymbol{u}_{\mathrm{f}}^{t}+h\left(f_{\mathrm{MPM}}^{t}+\mathbf{M}_{\mathrm{f}} \boldsymbol{g}+\mathrm{D}_{\mathrm{f}} \boldsymbol{u}_{\mathrm{s}}^{*}-\mathrm{V}_{\mathrm{f}} \mathrm{G}_{\mathrm{cf}} \boldsymbol{p}\right)
$$

Remark. Directly interpolating the drag forces onto the grid can introduce poor conditioning due to the interpolation matrix [Yue et al. 2018]. To avoid this issue, we instead interpolate the drag coefficients onto the grid, and then use them to recompute a gridbased drag force and apply it to the liquid. This choice makes the drag matrix diagonal and avoids conditioning issues, without introducing apparent visual artifacts. Yue et al. [2018] took a similar approach in the context of matching granular flow velocities solved with different models.

Semi-Analytic Plastic Flow. A Herschel-Bulkley liquid will yield and undergo plastic flow once its shear stress exceeds its yield stress, leading to a decrease of the shear stress. This fact must be considered when computing the Jacobian of the shear force, which necessitates the differentiability of the equation for plastic flow. However, prior work on Herschel-Bulkley liquids [Yue et al. 2015] computed the plastic flow with bisection, making the process non-differentiable. Fortunately, we found that the plastic flow for Herschel-Bulkley liquids can in fact be computed analytically, as described below.

In the following discussion, we use a bar to denote volumepreserving variables, e.g., the volume-preserving left Cauchy-Green strain is $\overline{\mathbf{b}}^{E} \equiv J^{-2 / d} \mathbf{b}^{E}$. Similar to prior work [Yue et al. 2015], we first compute an intermediate state for the updated normalized left Cauchy-Green strain as $\overline{\mathbf{b}}^{E, *}=\overline{\mathbf{f}} \overline{\mathbf{b}}^{E} \overline{\mathbf{f}}^{T}$ that accounts for the elastic deformation in (S12) (refer to supplemental §S1), where $\mathbf{f} \equiv \mathbf{I}_{d}+h \nabla \boldsymbol{u}_{\mathrm{f}}^{t}$ is the increment of the deformation gradient. The updated shear stress is then denoted as $\mathbf{s}^{*} \equiv \mu \operatorname{dev} \overline{\mathbf{b}}^{E, *}$ and its norm as $s^{*} \equiv\left\|\mathbf{s}^{*}\right\|$.

Once the plastic flow occurs, according to the von Mises yield condition (S11) (refer to supplemental §S1), we can update the norm $s^{*}$ as (the detailed derivation is given in supplemental §S9)

$$
s^{t+1}= \begin{cases}\left(s^{*}-\tilde{\sigma}_{Y}\right) e^{-\frac{2 \hat{\mu}}{\eta} h}+\tilde{\sigma}_{Y} & n=1 \\ {\left[\left(s^{*}-\tilde{\sigma}_{Y}\right)^{\frac{n-1}{n}}-2 \hat{\mu} h\left(1-\frac{1}{n}\right) \eta^{-\frac{1}{n}}\right]^{\frac{n}{n-1}}+\tilde{\sigma}_{Y}} & n \neq 1\end{cases}
$$

where $\hat{\mu} \equiv \frac{\mu}{3} \operatorname{tr} \overline{\mathbf{b}}^{E, *}, \tilde{\sigma}_{Y} \equiv \sqrt{\frac{2}{3}} \sigma_{Y}, \sigma_{Y}$ is the yield stress, $\eta$ is the flow consistency index, and $n$ is the flow behavior index. We can then recover the volume-preserving Cauchy-Green strain after plastic flow as $\overline{\mathbf{b}}^{E, t+1}=\frac{s^{t+1}}{s^{t}} \operatorname{dev}\left(\overline{\mathbf{b}}^{E, *}\right)+\hat{\mu} \mathbf{I}_{3}$.

The key benefit of (37) is that plastic flow becomes differentiable over $s^{*}$ and $\hat{\mu}$, making the shear stress differentiable. Hence we can compute the Jacobian matrix $\mathbf{H}_{\mathrm{f}}$ of the shear force (details in supplemental §S11) and implicitly integrate the shear stress with plastic flow considered. 


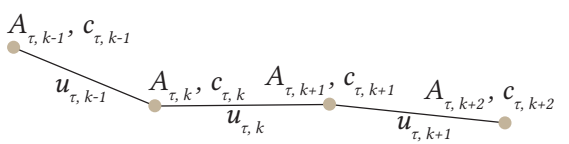

Fig. 15. A strand with staggered discrete flow variables: the cross-sectional area $A_{\tau}$ and the reduced elastic Cauchy-Green strain $c_{\tau}$ are defined on vertices, and the flow velocity $u_{\tau}$ is defined on edges.

Pressure Solve. After integrating the shear stress, we compute the pressure $\boldsymbol{p}$ using

$$
\mathrm{C}_{p} \boldsymbol{p}=\mathbf{K}_{c}^{-1} \boldsymbol{p}^{t}+h \mathrm{~V}_{\mathrm{c}}^{-1} \mathrm{G}_{\mathrm{w}, \mathrm{cn}}^{\mathrm{T}} \mathbf{V}_{\mathrm{s}}\left(\boldsymbol{u}_{\mathrm{s}}^{*}-\mathrm{W}_{\mathrm{fn}} \boldsymbol{u}_{\mathrm{f}}^{*}\right)+h \mathrm{G}_{\mathrm{cf}}^{\mathrm{T}} \boldsymbol{u}_{\mathrm{f}}^{*}
$$

where

$$
\mathrm{C}_{p}=\mathrm{K}_{c}^{-1}+h^{2} \mathrm{G}_{\mathrm{cf}}^{\mathrm{T}} \hat{\mathrm{C}}_{\mathrm{f}}^{-1} \mathbf{V}_{\mathrm{f}} \mathrm{G}_{\mathrm{cf}}
$$

the diagonal matrix $\mathrm{K}_{c}$ has $i$-th entry $\mathrm{K}_{c, i} \equiv \frac{\kappa}{2}\left(J_{i}^{E}+\frac{1}{J_{i}^{E}}\right)$, and $\hat{\mathrm{C}}_{\mathrm{f}}^{i}$ is a diagonal matrix to approximate $\mathrm{C}_{\mathrm{f}}$, i.e. $\hat{\mathrm{C}}_{f, k l}^{i}=\mathrm{C}_{f, k l}^{i} \delta_{k l}$. To preserve the symmetry of the pressure equation (38), we adopt explicit integration for the volume fraction change in (18b). We did not observe any instabilities resulting from this choice.

Surface Flow. We discretize the spatial derivatives in (2) with finite differences (Figure 15). At each time step we first advect both velocity $u_{\tau}$ and cross-sectional area $A_{\tau}$ with backtracing [Stam 1999]. We then integrate the remaining terms in (2) and update the cross-sectional area $A_{\tau}$ by integrating the divergence of velocity in (7). For the area at vertex $k$ we have

$$
A_{\tau, k}^{t+1}=A_{\tau, k}^{*} \exp \left(-h \frac{u_{\tau, k}-u_{\tau, k-1}}{l_{k}}\right)
$$

where $A_{\tau, k}^{*}$ is the area after advection, and $l_{k}$ is the Voronoi length of vertex $k$. Finally we update the Cauchy strain $c_{\tau}^{E}$ by discretizing the spatial derivatives in (3) with finite differences, which produces a nonlinear equation that we solve via bisection.

Contact Handling. Similar to prior work [Kaufman et al. 2014], we loop over all contacts during the collision solve, updating each contact while the remaining contacts are fixed, in a Gauss-Seidellike manner. By defining $\mathbf{E}$ as the tranformation from world space to contact space, the equation used to update the $k$-th contact is

$$
\boldsymbol{u}_{\mathrm{s}}^{t+1}=\boldsymbol{u}_{\mathrm{s}}^{\dagger}+\mathrm{C}_{\mathrm{s}}^{-1}\left(\mathrm{E}_{k} \boldsymbol{r}_{s, k}+\overline{\mathrm{E}}_{k} \overline{\boldsymbol{r}}_{s, k}\right),
$$

where $\overline{\mathrm{E}}_{k}$ and $\overline{\boldsymbol{r}}_{s, k}$ are respectively the complement matrix and vector formed by zeroing out the columns and entries used for $\mathrm{E}_{k}$ and $\boldsymbol{r}_{s, k}$.

In the following we define the future relative velocity at the contact as $\boldsymbol{v} \equiv \mathbf{E}^{T} \boldsymbol{u}_{\mathrm{s}}$. Multiplying by $\mathbf{E}^{T}$ on both sides of (41) gives

$$
\boldsymbol{v}^{t+1}=\mathrm{E}^{T}\left(\boldsymbol{u}_{\mathrm{s}}^{\dagger}+\mathrm{C}_{\mathrm{s}}^{-1} \mathrm{E}\left(\boldsymbol{r}+\boldsymbol{r}_{\mathrm{A}}\right)\right) .
$$

Above, we have decomposed the total contact impulse $\boldsymbol{r}_{s}$ into the unknown repulsive collision impulse $r$ and the known cohesive impulse $\boldsymbol{r}_{\mathrm{A}} \in \mathbb{R}^{3 k \times 1}$. The latter is a vector composed of cohesive forces in the normal directions of all contacts.

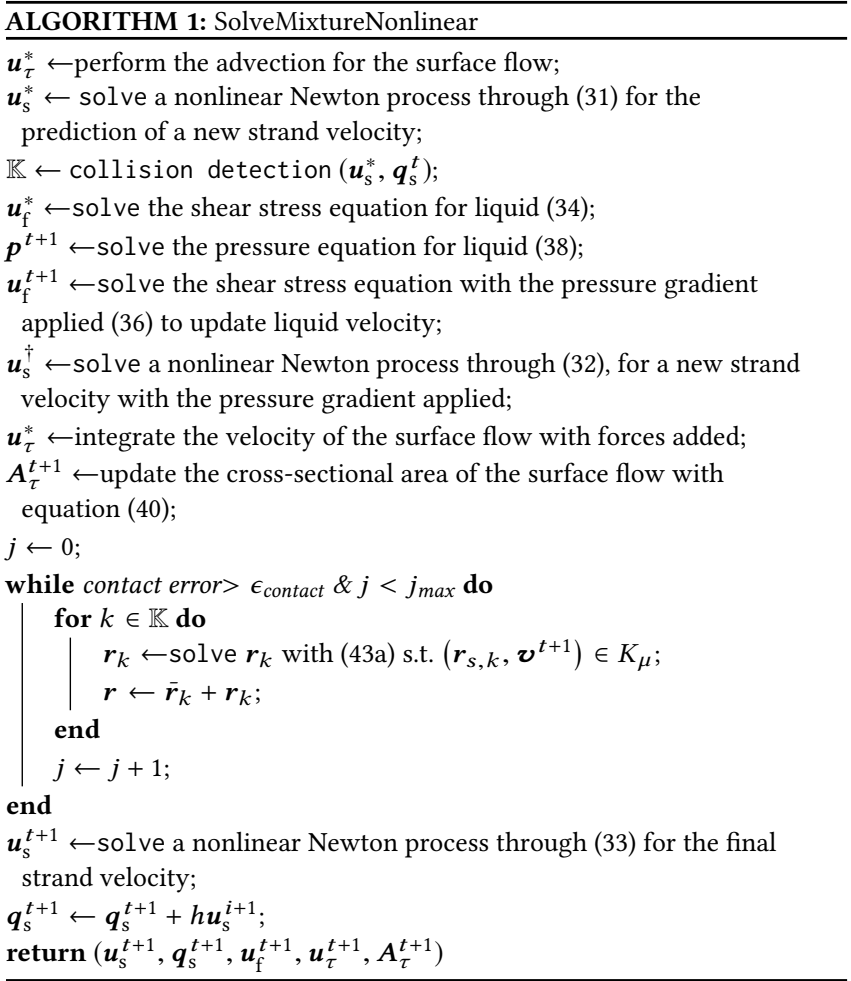

We can then reformulate the equations above as a second-order Coulomb cone problem (SOCCP) given by

$$
\begin{aligned}
& \mathrm{S} \boldsymbol{r}=\boldsymbol{v}-\mathrm{E}^{T} \boldsymbol{u}_{\mathrm{s}}^{\dagger}-\mathrm{S} \boldsymbol{r}_{\mathrm{A}}, \\
& \forall k,\left(\boldsymbol{r}_{s, k}, \boldsymbol{v}_{k}\right) \in K_{\mu}
\end{aligned}
$$

where $\mathrm{S}=\mathrm{E}^{\mathrm{T}} \mathbf{C}_{\mathrm{s}}{ }^{-1} \mathrm{E}$ is the Delassus operator [Bertails-Descoubes et al. 2011]. We solve this with a hybrid Gauss-Seidel solver [Daviet et al. 2011]. Compared with the equations for a standard SOCCP (see, e.g., [Daviet et al. 2011; Kaufman et al. 2014]), (43a) has a new cohesive term $\mathrm{Sr}_{\mathrm{A}}$ on the right hand side.

\subsection{Algorithm}

We summarize our resulting nonlinear mixture solver in Algorithm 1. For advection and mapping velocities between particles and the grid, we adopt the affine particle-in-cell (APIC) [Jiang et al. 2015] method and the moving least-squares material point method (MLSMPM) [Hu et al. 2018] for their simplicity and efficacy. As noted earlier, we rely on an underlying staggered (MAC) grid, similar to augmented MPM [Stomakhin et al. 2014]. We list the operations in a single step of our complete algorithm in supplemental §S12.

\section{RESULTS}

We divide our results into two classes: $i$ ) a group of didactic cases designed to validate individual components of our framework and ii) a set of more general scenarios of Herschel-Bulkley fluid interaction with strands that demonstrate the diversity of practical effects that can be achieved by our system. Details of how we reconstruct the liquid surface for rendering can be found in supplemental §S13. A 
Table 2. Timings and storage statistics. The timings are averaged over all the steps or frames of one example. Each frame is $1 / 30$ s. We adopt a time step of $1 \times 10^{-3}$ s for all examples, except for Soba with Oyster Sauce where we use a time step of $4 \times 10^{-4}$ s for more accurate collision detection. The total simulation time is the sum of time spent on the strands and liquid. The full lengths of animations vary between $4 \sim 8 \mathrm{~s}$.

\begin{tabular}{r|cccccccccc}
\hline Examples & \multicolumn{2}{|c}{ sec./step } & \multicolumn{2}{c}{ min./frame } & \multicolumn{2}{c}{ hour/anim. sec. } & \# particles & \# vertices & \multicolumn{2}{c}{ grid size } \\
strand & liquid & strand & liquid & strand & liquid & (max) & of strands & max dimensions & $\Delta x(\mathrm{~cm})$ \\
\hline Shaking a Hairball & 3.0 & 122.4 & 1.7 & 68.0 & 0.8 & 34.0 & $12.3 \mathrm{M}$ & $46.1 \mathrm{~K}$ & $120 \times 128 \times 120$ & 0.5 \\
Splashing Paint & 14.9 & 36.1 & 8.3 & 20.1 & 4.1 & 10.0 & $2.1 \mathrm{M}$ & $119.4 \mathrm{~K}$ & $328 \times 424 \times 328$ & 0.5 \\
Chocolate "Dog" & 20.2 & 57.3 & 11.2 & 31.8 & 5.6 & 15.9 & $3.0 \mathrm{M}$ & $688.1 \mathrm{~K}$ & $192 \times 272 \times 680$ & 0.75 \\
Soba with Oyster Sauce & 32.4 & 9.3 & 45 & 12.9 & 22.5 & 6.5 & $492.7 \mathrm{~K}$ & $97.0 \mathrm{~K}$ & $128 \times 80 \times 136$ & 0.375 \\
\hline
\end{tabular}

summary and discussion of the physics parameters used throughout this paper can be found in supplemental §S14.

\subsection{Didactic Examples}

Varying Volume Fraction. To show the importance of the volume fraction term in the pressure equation, we compare simulations of liquid flowing through hair with and without the volume fraction term used when solving the pressure equation (Fig. 7). For ease of comparison drag is disabled in this scene.

Buoyancy. Our method can correctly handle materials with different mass densities, which is not considered in prior work on liquid-hair coupling [Fei et al. 2017]. In Fig. 6 we demonstrate the buoyancy behavior of hairs with different mass densities in water.

Drag Force. In Fig. 9 we compare our material-specific drag coefficient against a constant drag coefficient for distinct liquids falling onto strands. In Fig. 14 we use the same scenario to compare the maximal (unitless) Courant number (calculated with $\frac{u_{\max } h}{\Delta x}$ where $u_{\max }$ is the maximal velocity across the whole domain, $h$ is the time step, and $\Delta x$ is the cell size) between different integration schemes. Compared with using an explicit integrator for the shear stress, or for both shear and pressure, our semi-implicit integrator is stable for both compressible shaving cream and incompressible drilling mud, and can handle more or less viscous liquids with moderate time steps (Courant number up to approximately 1.24).

Droplet Dripping. In Fig. 2 large droplets of various liquids flow down thin strands, demonstrating the variety of material-dependent behavior that we capture. In Fig. 5, we construct a similar scenario with a large, heavy tetrachloroethene droplet (mass density $1.622 \mathrm{~g} / \mathrm{cm}$ ). When such a droplet flows on a thin strand, the flow can dramatically affect the strand's momentum. With the explicit inertia transfer method of prior work [Fei et al. 2017], a huge correction will cause instability. On the other hand, our improved method can stably integrate the extra strand momentum induced by the large droplet.

High-Speed Rotation. To further demonstrate the behavior of surface flows for liquids with high viscosity or yield stress, we rapidly rotate strands with droplets of various materials on them (Fig. 4). We observe that the mud and paint are flung out quickly after the simulation starts, while the milk cream starts to move only after the centrifugal force exceeds its yield stress. We also observed that the paint and milk cream flow more easily as their velocities are increased, demonstrating a shear-thinning behavior.

Cohesion and coalescence. In Fig. 10, two strands initially hang vertically with a distance of $0.01 \mathrm{~cm}$ between their centerlines. We then gradually separate them. The milk cream between the two strands forms a strong cohesive force. Comparing our constraint-based model with the penalty-based model, the latter exhibits tunneling and locking artifacts when simulating the cohesion of strongly nonNewtonian materials.

Friction with Various Materials. To demonstrate how the liquid material affects friction between strands, we simulate two bundles of strands covered with cream and mud, respectively (figure 13). Since the cream has higher viscosity and yield stress, the strands covered in cream move less readily compared with those covered in mud.

\subsection{Large-Scale Examples}

Shaking a Hairball. To illustrate the cohesive and frictional behavior of wet hairs, we simulate a hair-covered ball lifting out of a mud pool and shaking (Fig. 16a). When the motion stops, the hairs stick and tangle as expected.

Splashing Paint. Oil paint is another familiar shear-dependent fluid. We simulate the interaction between the brush bristles and a pool of colorful paint (Fig. 16b). In contrast to examples from earlier work (e.g., [Chen et al. 2015; Zhu et al. 2015]) in which a brush moves slowly across paper and is one-way coupled with the paint, we show a brush that is two-way coupled with the paint during violent and rapid rotation, which causes large and dynamic splashing of the paint.

Chocolate "Dog". Recent work on wet hair [Fei et al. 2017] simulated the process of pouring water over a rotating shaggy-haired cylinder, mimicking mammal shaking behavior [Dickerson et al. 2012]. To contrast water against more complex liquids, we revisit this scenario replacing the water with molten chocolate. Before the cylinder begins rotating, the falling chocolate forms thin sheets and tendrils; later, it separates into many chunky pieces. At the end of the rotation, much of the chocolate has adhered to the clumped hairs, as would be expected (Fig. 16c).

Soba with Oyster Sauce. Moving beyond hair strands, our method can simulate the coupling between a plate of Soba (buckwheat) noodle and thick oyster sauce. As the fork is pulled up, due to the frictional effect induced by the strong viscosity of the sauce, the noodles naturally stick to the liquid and each other (Fig. 16d).

\subsection{Performance Numbers}

The timing data for our large scale examples is presented in Table 2, measured on a workstation with 2 sockets of Intel Xeon E5-2620 v3 


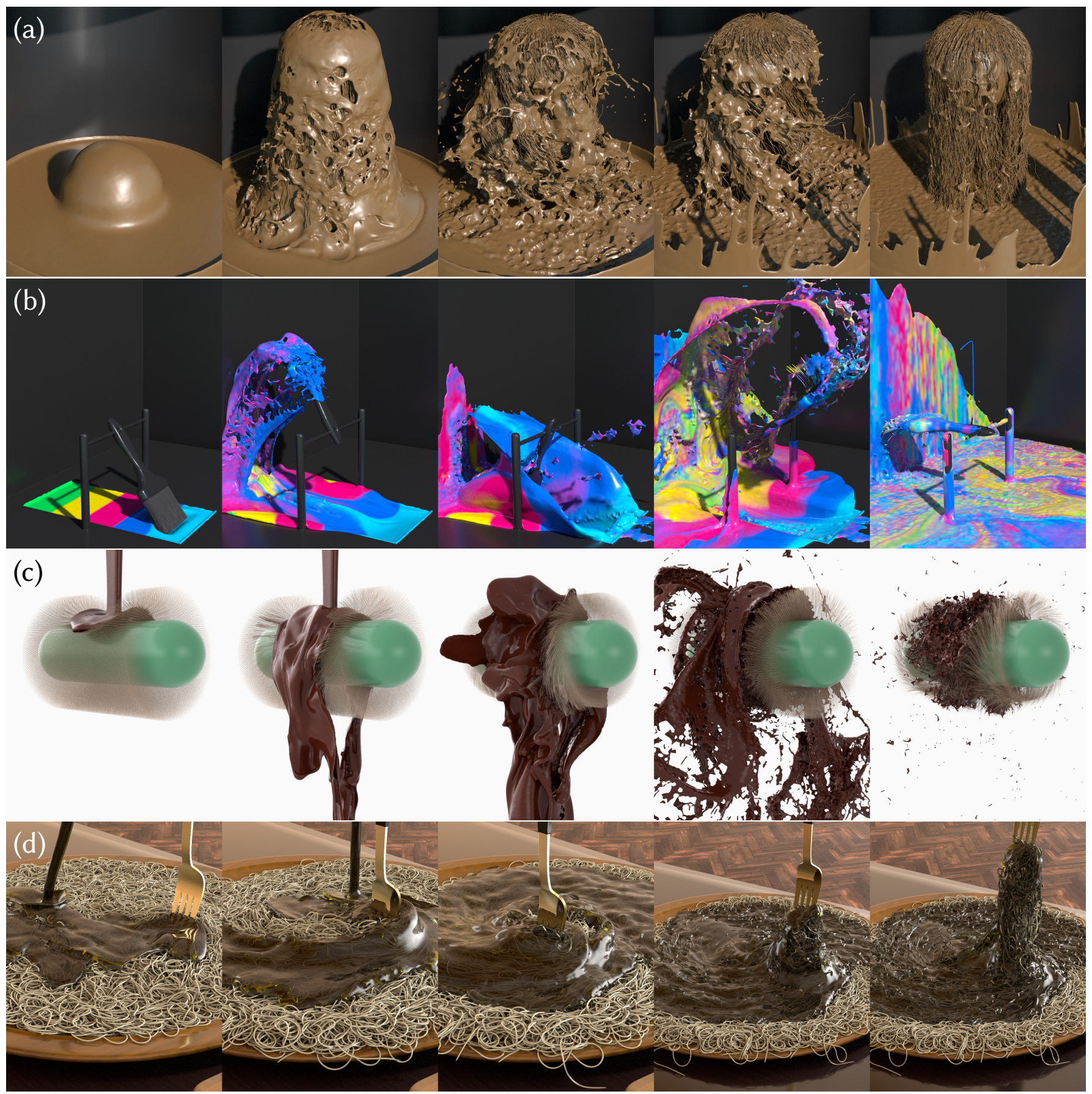

Fig. 16. (a) Shaking a Hairball. A ball rises out of mud and shakes its hairs to throw off the mud. (b) Splashing the Paint. A rapidly rotating paint brush splashes oil paint everywhere. (c) Chocolate "dog". Melted chocolate is poured onto a fluffy cylinder, which tries to shake the chocolate off by mimicking the mammal-shaking behavior. (d) Soba with Oyster Sauce. Oyster sauce is poured onto a plate of soba noodles, while a fork is used to stir and pull the noodles.

CPUs running at $2.4 \mathrm{GHz}$, each of which has 6 cores. We also provide a detailed breakdown in Figure 17 for the Chocolate "dog" example.

\section{DISCUSSIONS AND LIMITATIONS}

We have presented a multi-scale framework that can couple strands with diverse shear-dependent liquids. The results in this paper cover 

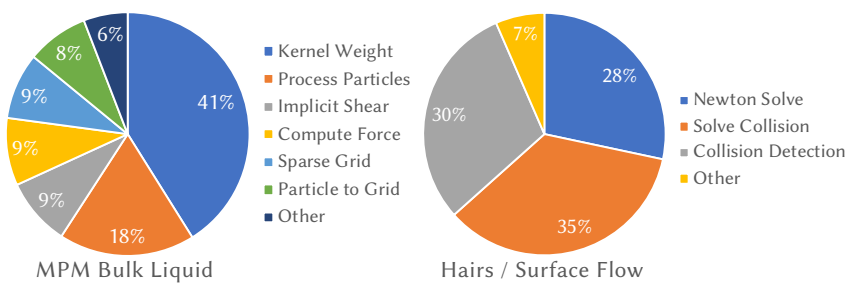

Fig. 17. Timing Statistics for the Chocolate "Dog"

a wide range of materials, including incompressible or compressible, and Newtonian or non-Newtonian fluids.

Several of our developments benefit the coupling of strands to fluids in general, be they Newtonian or non-Newtonian. The pressure equation (18a) accommodates various constitutive models [Jiang et al. 2016]. The momentum equation (9) of strands is agnostic to the fluid model. Indeed, (9) applies to any solid (not just strands) with surface flow, e.g., raindrops sliding over a glass pane. Di Felice's drag formula is equally applicable to both Newtonian and non-Newtonian fluid. The same is true of the boundary condition for the consistency between surface flow and bulk liquid (§3.4.3), and our extension of the second-order Coulomb cone to consider cohesion (§3.5).

Some of our other contributions are naturally more specific to shear-dependent fluids. The reduced Cauchy-Green strain and its dynamics (§3.1) are exclusive to shear-dependent surface flow. Aspects of the cohesive force and the semi-analytical formulation of the plastic flow (37) are only meaningful for Herschel-Bulkley fluids. Although Di Felice's drag formula is a comprehensive treatment, Renaud's drag coefficient is not, and is only required for coupling solids with non-Newtonian fluids [Renaud et al. 2004; Tabuteau et al 2007].

Aside from the physical correctness, we also care about the impact of different components on the visual look. In supplemental §S15 we discuss the importance of all the components that need to be implemented.

\subsection{Limitations}

Our framework still has a few limitations imposed by our assumptions, numerical methods, surface reconstructors, or selected experiments.

In the reduced surface flow model, surface tension in the longitudinal direction is neglected for ease of computation, and thus we cannot maintain the correct contact angle between the reduced surface flow and the strand. Also, large droplets that break the cylindrical assumption cannot be faithfully captured. We have not considered the fact that some strands may have anisotropic crosssections. For some materials (e.g., milk cream), the surface liquid is slow in the absence of drastic strand motion, and hence the computation could be further simplified for better performance. Our liquid capturing process is purely geometric, and thus the liquid can exhibit some popping artifacts when the simulation replays slowly.

The pressure equation can be imprecise when the strands are compressible (e.g., rubber bands). We have not considered coupling strands with materials whose plastic flows are pressure-dependent, such as snow, sand, or rubber. The pressure solved in our staggered integration approach may not be consistent with the future velocity of the strands, although we have not observed visible artifacts caused by this fact. Similar to prior work [Stomakhin et al. 2014], our bulk fluid model splits the integration of pressure from shear stress, which may affect the accuracy of the free surface [Larionov et al. 2017].

Due to the limited resolution of the fluid grid, the drag force can affect the liquid motion over a wider region than expected if the grid resolution is insufficient. Increasing the resolution of the grid or using an adaptive grid [Gao et al. 2017] may alleviate this problem.

In our supplemental video, we show several examples of realworld footage to give foundation to the visual results. We have also considered carrying out carefully registered comparisons between virtual and laboratory experiments, and found this to be challenging and a major undertaking in its own right. Tasks that must be successfully achieved would include (1) design and control of a physical apparatus to carry out laboratory experiments, (2) measuring and matching constitutive properties and other physical parameters, (3) registering virtual and laboratory initial and boundary conditions, including matching hair geometry, temperatures (and thus in turn the liquid viscosity), injection rates, etc. To keep a focused scope of this paper, we believe that the parameter capture and the setup of experimental apparatus deserve a delicate development-a worthy topic for future research.

Some limitations are inherited from the sub-components adapted to build our framework. Augmented MPM spends more time computing (staggered) kernel weights than regular MPM. The hybrid iterative solver used for the SOCCP is not strictly guaranteed to converge, which may cause some penetrations (e.g., in Soba with Oyster Sauce, there are some penetrations between the noodle and the plate, which, however, are not observable in the video). This is caused by the non-convergence of the SOCCP solver, which is a known problem for such a Gauss-Seidel solver. Neither is the stability of the elastic rods guaranteed: when two consecutive rod elements are bent to (almost) 180-degrees, the discrete curvature becomes (near-)singular, and the bending energy would rise to infinity. This issue can occur in practice when a large drag force reshapes the strands into a problematic configuration.

The visual realism depends on not only the simulated dynamics but also the surface reconstruction. We adopted the standard particle surfacing method implemented in Houdini [2019] for all our examples. Although widely applied to Newtonian liquids, this method largely ignores the particles' deformation history, such that thin sheets or tendrils may appear as separated droplets during reconstruction, thus resulting in the clumpy (particle-like) look in some cases. Increasing the number of liquid particles may partially alleviate this issue, but would dramatically increase the simulation cost. We leave the non-trivial task of developing a deformationaware reconstruction method as future work, while focusing on the simulation model in this paper.

Moreover, developing accurate shaders for rendering such nonNewtonian liquids can be difficult. For example, we tried using ketchup in Soba with Oyster Sauce but did not successfully obtain a ketchup shader with a realistic visual appearance. We believe many of the limitations described above can provide exciting fodder for future research.

ACM Trans. Graph., Vol. 38, No. 6, Article 190. Publication date: November 2019. 


\section{ACKNOWLEDGMENTS}

This work was supported in part by the National Science Foundation under Grant Nos.: 1717178, 1319483, CAREER-1453101, the Natural Sciences and Engineering Research Council of Canada under Grant No. RGPIN-04360-2014, SoftBank Group, Pixar, Adobe, and SideFX. We also thank Florence Bertails-Descoubes, Theodore Kim, and Peter Yichen Chen for inspiring discussions, Henrique Maia for his contribution to the voiceover, Ryan Goldade for his assistence on surface reconstruction, Xiaowei Tan for his advice on the rendering, Adela Cheng for her help on the real footage (refer to our supplemental video), Robert Lane for his help on the computational devices, and Cristin Barghiel for his continued generous contribution of Houdini licenses. We would also like to thank Gilles Daviet, Jean-Marie Aubry, Yonghao Yue, Breannan Smith, Danny Kaufman, Xinxin Zhang, Mark Leone, Miklos Bergou and Susan Howard for the sharing of their code. In addition, we would like to thank the anonymous reviewers for their insightful comments.

\section{REFERENCES}

A Acharya, RA Mashelkar, and J Ulbrecht. 1976. Flow of inelastic and viscoelastic fluids past a sphere. Rheologica Acta 15, 9 (1976), 454-470.

Neha Agarwal and RP Chhabra. 2007. Settling velocity of cubes in Newtonian and power law liquids. Powder Technology 178, 1 (2007), 17-21.

T B Anderson and Roy Jackson. 1967. Fluid mechanical description of fluidized beds Equations of motion. Industrial \& Engineering Chemistry Fundamentals 6, 4 (1967), 527-539.

Nicolas Andreini, Gaël Epely-Chauvin, and Christophe Ancey. 2012. Internal dynamics of Newtonian and viscoplastic fluid avalanches down a sloping bed. Physics of Fluids 24, 5 (2012), 053101

Hesam Anvari Ardakani, Evan Mitsoulis, and Savvas G Hatzikiriakos. 2014. Capillary flow of milk chocolate. Journal of Non-Newtonian Fluid Mechanics 210 (2014), 56-65.

Larry Armijo. 1966. Minimization of functions having Lipschitz continuous first partial derivatives. Pacific 7. Math. 16, 1 (1966), 1-3.

Teodor M Atanackovic and Ardéshir Guran. 2012. Theory of elasticity for scientists and engineers. Springer Science \& Business Media.

DD Atapattu, RP Chhabra, and PHT Uhlherr. 1995. Creeping sphere motion in HerschelBulkley fluids: flow field and drag. Journal of Non-Newtonian Fluid Mechanics 59, 2-3 (1995), 245-265.

Omri Azencot, Orestis Vantzos, Max Wardetzky, Martin Rumpf, and Mirela Ben-Chen 2015. Functional thin films on surfaces. In Proceedings of the 14th ACM SIGGRAPH/Eurographics Symposium on Computer Animation (SCA '15). ACM, 137-146.

Samila Bandara and Kenichi Soga. 2015. Coupling of soil deformation and pore fluid flow using material point method. Computers and Geotechnics 63 (2015), 199-214.

M Beaulne and E Mitsoulis. 1997. Creeping motion of a sphere in tubes filled with Herschel-Bulkley fluids. Journal of Non-Newtonian Fluid Mechanics 72, 1 (1997), 55-71.

A Bedford and D S Drumheller. 1983. Theories of immiscible and structured mixtures International fournal of Engineering Science 21, 8 (1983), 863-960.

Miklós Bergou, Basile Audoly, Etienne Vouga, Max Wardetzky, and Eitan Grinspun. 2010. Discrete viscous threads. ACM Transactions on Graphics (TOG) 29, 4 (2010), 116.

Miklós Bergou, Max Wardetzky, Stephen Robinson, Basile Audoly, and Eitan Grinspun. 2008. Discrete Elastic Rods. ACM Transactions on Graphics (TOG) 27, 3 (aug 2008), 63:1-63:12.

AN Beris, JA Tsamopoulos, RC Armstrong, and RA Brown. 1985. Creeping motion of a sphere through a Bingham plastic. Journal of Fluid Mechanics 158 (1985), 219-244.

Florence Bertails, Basile Audoly, Marie-Paule Cani, Bernard Querleux, Frédéric Leroy, and Jean-Luc Lévêque. 2006. Super-helices for predicting the dynamics of natural hair. ACM Transactions on Graphics (TOG) 25, 3 (2006), 1180-1187.

Florence Bertails, Sunil Hadap, Marie-Paule Cani, Ming Lin, Tae-Yong Kim, Steve Marschner, Kelly Ward, and Zoran Kačić-Alesić. 2008. Realistic hair simulation animation and rendering. In ACM SIGGRAPH 2008 Courses. ACM, 89.

Florence Bertails-Descoubes, Florent Cadoux, Gilles Daviet, and Vincent Acary. 2011. A nonsmooth Newton solver for capturing exact Coulomb friction in fiber assemblies. ACM Transactions on Graphics (TOG) 30, 1 (2011), 6

Bharat Bhushan, Guohua Wei, and Paul Haddad. 2005. Friction and wear studies of human hair and skin. Wear 259, 7-12 (2005), 1012-1021.

AA Bochkarev, PI Geshev, VI Polyakova, and NI Yavorskii. 2009. Formal rheological model of acrylic waterborne paints. Theoretical Foundations of Chemical Engineering
43, 1 (2009), 100-107.

Javier Bonet and Richard D Wood. 1997. Nonlinear continuum mechanics for finite element analysis. Cambridge university press.

Ronaldo I Borja. 2006. On the mechanical energy and effective stress in saturated and unsaturated porous continua. International fournal of Solids and Structures 43, 6 (2006), 1764-1786.

Percy Williams Bridgman. 1949a. Linear Compressions to $30,000 \mathrm{~kg} / \mathrm{cm}^{2}$, including Relatively Incompressible Substances. Proceedings of the American Academy of Arts and Sciences 77, 6 (1949), 189-234.

Percy Williams Bridgman. 1949b. The physics of high pressure. London: Bells and Sons.

Robert Bridson. 2015. Fluid simulation for computer graphics. CRC Press.

F Brochard and PG De Gennes. 1992. Shear-dependent slippage at a polymer/solid interface. Langmuir 8, 12 (1992), 3033-3037.

Philip Crosbie Carman. 1937. Fluid flow through granular beds. Transactions of the Institution of Chemical Engineers 15 (1937), 150-166.

Zhili Chen, Byungmoon Kim, Daichi Ito, and Huamin Wang. 2015. Wetbrush: GPUbased 3D painting simulation at the bristle level. ACM Transactions on Graphics (TOG) 34, 6 (2015), 200

Raj P Chhabra. 2006. Bubbles, drops, and particles in non-Newtonian fluids. CRC press.

RV Craster and OK Matar. 2009. Dynamics and stability of thin liquid films. Reviews of Modern Physics 81, 3 (2009), 1131.

Henry Philibert Gaspard Darcy. 1856. Dètermination des lois d'ècoulement de l'eau à travers le sable.

Gilles Daviet and Florence Bertails-Descoubes. 2016. A semi-implicit material point method for the continuum simulation of granular materials. ACM Transactions on Graphics (TOG) 35, 4 (2016), 102.

Gilles Daviet and Florence Bertails-Descoubes. 2017. Simulation of Drucker-Prager granular flows inside Newtonian fluids. (Feb. 2017). working paper or preprint.

Gilles Daviet, Florence Bertails-Descoubes, and Laurence Boissieux. 2011. A hybrid iterative solver for robustly capturing coulomb friction in hair dynamics. ACM Transactions on Graphics (TOG) 30, 6 (2011), 139.

Reint De Boer. 2012. Theory of porous media: highlights in historical development and current state. Springer Science \& Business Media.

Crispin Deul, Tassilo Kugelstadt, Marcel Weiler, and Jan Bender. 2018. Direct PositionBased Solver for Stiff Rods. Computer Graphics Forum 37, 6 (2018), 313-324.

R Di Felice. 1994. The voidage function for fluid-particle interaction systems. International fournal of Multiphase Flow 20, 1 (1994), 153-159.

Andrew K Dickerson, Zachary G Mills, and David L Hu. 2012. Wet mammals shake at tuned frequencies to dry. Fournal of the Royal Society Interface 9, 77 (2012), 3208-3218.

Sabri Ergun. 1952. Fluid flow through packed columns. Chemical Engineering Progress 48 (1952), 89-94.

Yu Fang, Minchen Li, Ming Gao, and Chenfanfu Jiang. 2019. Silly Rubber: An Implicit Material Point Method for Simulating Non-equilibrated Viscoelastic and Elastoplastic Solids. ACM Transactions on Graphics (TOG) 38, 4 (2019).

Yun (Raymond) Fei, Christopher Batty, Eitan Grinspun, and Changxi Zheng. 2018. A multi-scale model for simulating liquid-fabric interactions. ACM Transactions on Graphics (TOG) 37, 4 (2018), 51.

Yun (Raymond) Fei, Henrique Teles Maia, Christopher Batty, Changxi Zheng, and Eitan Grinspun. 2017. A Multi-scale Model for Simulating Liquid-hair Interactions. ACM Transactions on Graphics (TOG) 36, 4, Article 56 (July 2017), 17 pages.

PH Forchheimer. 1901. Wasserbewegung durch boden. Zeitz. Ver. Duetch Ing. 45 (1901), $1782-1788$.

Chuyuan Fu, Qi Guo, Theodore Gast, Chenfanfu Jiang, and Joseph Teran. 2017. A Polynomial Particle-In-Cell Method. ACM Transactions on Graphics (TOG) 36, 6 (2017), 222.

Ming Gao, Andre Pradhana, Xuchen Han, Oi Guo, Grant Kot, Eftychios Sifakis, and Chenfanfu Jiang. 2018a. Animating fluid sediment mixture in particle-laden flows. ACM Transactions on Graphics (TOG) 37, 4 (2018), 149.

Ming Gao, Andre Pradhana Tampubolon, Chenfanfu Jiang, and Eftychios Sifakis. 2017. An adaptive generalized interpolation material point method for simulating elastoplastic materials. ACM Transactions on Graphics (TOG) 36, 6 (2017), 223.

Ming Gao, Xinlei Wang, Kui Wu, Andre Pradhana Tampubolon, Eftychios Sifakis, Cem Yuksel, and Chenfanfu Jiang. 2018b. GPU Optimization of Material Point Methods. ACM Transactions on Graphics (TOG) 37, 6 (2018).

J Gaume, T Gast, J Teran, A van Herwijnen, and C Jiang. 2018. Dynamic anticrack propagation in snow. Nature Communications 9, 1 (2018), 3047.

Jean-Frédéric Gerbeau and Benoît Perthame. 2001. Derivation of Viscous Saint-Venant System for Laminar Shallow Water; Numerical Validation. Discrete and Continuous Dynamical Systems-Series B 1, 1 (2001), 89-102.

Galen Gornowicz and Silviu Borac. 2015. Efficient and stable approach to elasticity and collisions for hair animation. In Proceedings of the 2015 Symposium on Digital Production. ACM, 41-49.

Mireille Grégoire and Elmar Schömer. 2007. Interactive simulation of one-dimensional flexible parts. Computer-Aided Design 39, 8 (2007), 694-707. 
Qi Guo, Xuchen Han, Chuyuan Fu, Theodore Gast, Rasmus Tamstorf, and Joseph Teran. 2018. A material point method for thin shells with frictional contact. ACM Transactions on Graphics (TOG) 37, 4 (2018), 147.

Sunil Hadap, Marie-Paule Cani, Florence Bertails, Ming Lin, Kelly Ward, Stephen Marschner, Tae-Yong Kim, and Zoran Kacic-Alesic. 2007. Strands and hair - Modeling, simulation and rendering. In SIGGRAPH Courses. 1-150.

Sunil Hadap and Nadia Magnenat-Thalmann. 2001. Modeling dynamic hair as a continuum. Computer Graphics Forum 20, 3 (2001), 329-338.

Dongsoo Han and Takahiro Harada. 2013. Tridiagonal matrix formulation for inextensible hair strand simulation. In Proceedings of the Workshop on Virtual Reality Interaction and Physical Simulation. The Eurographics Association.

Fathi Hassine. 2013. A review of depth-averaged models for dry granular flows: SavageHutter and $\mu(I)$-rheology. arXiv preprint arXiv:1303.2838 (2013).

Winslow H Herschel and Ronald Bulkley. 1926. Konsistenzmessungen von gummibenzollösungen. Colloid \& Polymer Science 39, 4 (1926), 291-300.

JE Hilton, LR Mason, and PW Cleary. 2010. Dynamics of gas-solid fluidised beds with non-spherical particle geometry. Chemical Engineering Science 65, 5 (2010), 1584-1596.

Damien Hinsinger, Fabrice Neyret, and Marie-Paule Cani. 2002. Interactive animation of ocean waves. In Proceedings of the 2002 ACM SIGGRAPH/Eurographics Symposium on Computer animation (SCA '02). ACM, 161-166.

Yuanming Hu, Yu Fang, Ziheng Ge, Ziyin Qu, Yixin Zhu, Andre Pradhana, and Chenfanfu Jiang. 2018. A moving least squares material point method with displacement discontinuity and two-way rigid body coupling. ACM Transactions on Graphics (TOG) 37,4 (2018), 150

D Iesan. 1994. On the theory of mixtures of elastic solids. Fournal of Elasticity 35, 1-3 (1994), 251-268.

D Ieşan and L Nappa. 2008. On the theory of viscoelastic mixtures and stability. Mathematics and Mechanics of Solids 13, 1 (2008), 55-80.

Markus Ihmsen, Jens Cornelis, Barbara Solenthaler, Christopher Horvath, and Matthias Teschner. 2014. Implicit incompressible SPH. IEEE Transactions on Visualization and Computer Graphics 20, 3 (2014), 426-435.

Ioan R Ionescu. 2010. Onset and dynamic shallow flow of a viscoplastic fluid on a plane slope. Journal of Non-Newtonian Fluid Mechanics 165, 19-20 (2010), 1328-1341.

Ioan R Ionescu. 2013a. Augmented Lagrangian for shallow viscoplastic flow with topography. 7. Comput. Phys. 242 (2013), 544-560.

Ioan R Ionescu. 2013b. Viscoplastic shallow flow equations with topography. Fournal of Non-Newtonian Fluid Mechanics 193 (2013), 116-128.

Ioan R Ionescu and Oana Lupaşcu. 2016. Modeling shallow avalanche onset over complex basal topography. Advances in Computational Mathematics 42, 1 (2016), $5-26$.

Ioan R Ionescu, Anne Mangeney, François Bouchut, and Olivier Roche. 2015. Viscoplastic modeling of granular column collapse with pressure-dependent rheology. Fournal of Non-Newtonian Fluid Mechanics 219 (2015), 1-18.

Fridtjov Irgens. 2008. Continuum mechanics. Springer Science \& Business Media.

Fridtjov Irgens. 2014. Generalized Newtonian Fluids. Springer International Publishing Cham, 113-124. https://doi.org/10.1007/978-3-319-01053-3 6

M Khalid Jawed, Alyssa Novelia, and Oliver M O'Reilly. 2018. A primer on the kinematics of discrete elastic rods. Springer.

Michel Jean. 1999. The non-smooth contact dynamics method. Computer Methods in Applied Mechanics and Engineering 177, 3-4 (1999), 235-257.

Michel Jean, Vincent Acary, and Yann Monerie. 2001. Non-smooth contact dynamics approach of cohesive materials. Philosophical Transactions of the Royal Society of London. Series A: Mathematical, Physical and Engineering Sciences 359, 1789 (2001), 2497-2518.

Chenfanfu Jiang, Theodore Gast, and Joseph Teran. 2017. Anisotropic elastoplasticity for cloth, knit and hair frictional contact. ACM Transactions on Graphics (TOG) 36, 4 (2017), 152.

Chenfanfu Jiang, Craig Schroeder, Andrew Selle, Joseph Teran, and Alexey Stomakhin 2015. The affine particle-in-cell method. ACM Transactions on Graphics (TOG) 34, 4 (2015) 51.

Chenfanfu Jiang, Craig Schroeder, Joseph Teran, Alexey Stomakhin, and Andrew Selle 2016. The material point method for simulating continuum materials. In $A C M$ SIGGRAPH 2016 Courses. ACM, 24

Dirk Kadau, Guido Bartels, Lothar Brendel, and Dietrich E Wolf. 2002. Contact dynamics simulations of compacting cohesive granular systems. Computer Physics Communications 147, 1-2 (2002), 190-193.

Jonathan M. Kaldor, Doug L. James, and Steve Marschner. 2010. Efficient Yarn-based Cloth with Adaptive Contact Linearization. ACM Transactions on Graphics (TOG) 29, 4, Article 105 (July 2010), 10 pages.

Michael Kass and Gavin Miller. 1990. Rapid, stable fluid dynamics for computer graphics. In ACM SIGGRAPH Computer Graphics, Vol. 24. ACM, 49-57.

Danny M. Kaufman, Rasmus Tamstorf, Breannan Smith, Jean-Marie Aubry, and Eitan Grinspun. 2014. Adaptive Nonlinearity for Collisions in Complex Rod Assemblies. ACM Transactions on Graphics (TOG) 33, 4, Article 123 (July 2014), 12 pages.
VC Kelessidis, R Maglione, C Tsamantaki, and Y Aspirtakis. 2006. Optimal determination of rheological parameters for Herschel-Bulkley drilling fluids and impact on pressure drop, velocity profiles and penetration rates during drilling. Fournal of Petroleum Science and Engineering 53, 3-4 (2006), 203-224.

Gergely Klár, Theodore Gast, Andre Pradhana, Chuyuan Fu, Craig Schroeder, Chenfanfu Jiang, and Joseph Teran. 2016. Drucker-prager elastoplasticity for sand animation. ACM Transactions on Graphics (TOG) 35, 4 (2016), 103.

Tassilo Kugelstadt and Elmar Schömer. 2016. Position and orientation based Cosserat rods. In Proceedings of the ACM SIGGRAPH/Eurographics Symposium on Computer Animation. Eurographics Association, 169-178.

JAM Kuipers, KJ Van Duin, FPH Van Beckum, and Willibrordus Petrus Maria Van Swaaij. 1992. A numerical model of gas-fluidized beds. Chemical Engineering Science 47, 8 (1992), 1913-1924.

Hanna G Lagger, Thomas Breinlinger, Jan G Korvink, Michael Moseler, Alberto Di Renzo, Francesco Di Maio, and Claas Bierwisch. 2015. Influence of hydrodynamic drag model on shear stress in the simulation of magnetorheological fluids. Fournal of Non-Newtonian Fluid Mechanics 218 (2015), 16-26.

Horace Lamb. 1993. Hydrodynamics. Cambridge university press.

Egor Larionov, Christopher Batty, and Robert Bridson. 2017. Variational stokes: a unified pressure-viscosity solver for accurate viscous liquids. ACM Transactions on Graphics (TOG) 36, 4 (2017), 101

Minjae Lee, David Hyde, Michael Bao, and Ronald Fedkiw. 2019. A skinned tetrahedral mesh for hair animation and hair-water interaction. IEEE Transactions on Visualization and Computer Graphics 25, 3 (2019), 1449-1459.

Toon Lenaerts, Bart Adams, and Philip Dutré. 2008. Porous flow in particle-based fluid simulations. ACM Transactions on Graphics (TOG) 27, 3 (2008), 49.

Xingyue Li and Jidong Zhao. 2018. Dam-break of mixtures consisting of non-Newtonian liquids and granular particles. Powder Technology 338 (2018), 493-505.

Guoping Lian, Colin Thornton, and Michael J Adams. 1993. A theoretical study of the liquid bridge forces between two rigid spherical bodies. Fournal of Colloid and Interface Science 161, 1 (1993), 138-147.

Wei-Chin Lin. 2014. Coupling Hair with Smoothed Particle Hydrodynamics Fluids. In Workshop on Virtual Reality Interaction and Physical Simulation, Jan Bender, Christian Duriez, Fabrice Jaillet, and Gabriel Zachmann (Eds.). The Eurographics Association.

Wei-Chin Lin. 2015. Boundary handling and porous flow for fluid-hair interactions Computers \& Graphics 52 (2015), 33-42.

E Mauret and M Renaud. 1997. Transport phenomena in multi-particle systems-II. Proposed new model based on flow around submerged objects for sphere and fiber beds-transition between the capillary and particulate representations. Chemical Engineering Science 52, 11 (1997), 1819-1834.

Aleka McAdams, Andrew Selle, Kelly Ward, Eftychios Sifakis, and Joseph Teran. 2009. Detail preserving continuum simulation of straight hair. ACM Transactions on Graphics (TOG) 28, 3 (2009), 62.

R v Mises. 1913. Mechanik der festen Körper im plastisch-deformablen Zustand Nachrichten von der Gesellschaft der Wissenschaften zu Göttingen, MathematischPhysikalische Klasse 1913 (1913), 582-592.

Joe J Monaghan. 1994. Simulating free surface flows with SPH. f. Comput. Phys. 110, 2 (1994), 399-406.

Jean J Moreau. 1988. Unilateral contact and dry friction in finite freedom dynamics. In Nonsmooth Mechanics and Applications. Springer, 1-82.

Matthias Müller, David Charypar, and Markus Gross. 2003. Particle-based fluid simulation for interactive applications. In Proceedings of the 2003 ACM SIGGRAPH/Eurographics symposium on Computer animation. Eurographics Association, $154-159$

Matthias Müller, Tae-Yong Kim, and Nuttapong Chentanez. 2012. Fast simulation of inextensible hair and fur.. In Proceedings of the Workshop on Virtual Reality Interaction and Physical Simulation, Vol. 12.39-44.

Kentaro Nagasawa, Takayuki Suzuki, Ryohei Seto, Masato Okada, and Yonghao Yue. 2019. Mixing Sauces: A Viscosity Blending Model for Shear Thinning Fluids. ACM Transactions on Graphics (TOG) 38, 4 (2019).

Michael B Nielsen and Ole Østerby. 2013. A two-continua approach to Eulerian simulation of water spray. ACM Transactions on Graphics (TOG) 32, 4 (2013), 67.

Hilary Ockendon and John R Ockendon. 1995. Viscous flow. Vol. 13. Cambridge University Press.

Alexander Oron, Stephen H Davis, and S George Bankoff. 1997. Long-scale evolution of thin liquid films. Reviews of Modern Physics 69, 3 (1997), 931.

Saket Patkar and Parag Chaudhuri. 2013. Wetting of porous solids. IEEE Transactions on Visualization and Computer Graphics 19, 9 (2013), 1592-1604.

JRA Pearson and PMJ Tardy. 2002. Models for flow of non-Newtonian and complex fluids through porous media. Journal of Non-Newtonian Fluid Mechanics 102, 2 (2002), 447-473.

Charles S Peskin. 2002. The immersed boundary method. Acta numerica 11 (2002), 479-517

Lena Petrovic, Mark Henne, and John Anderson. 2005. Volumetric methods for simulation and rendering of hair. Pixar Animation Studios 2, 4 (2005). 
Remmelt Pit, Hubert Hervet, and Liliane Léger. 1999. Friction and slip of a simple liquid at a solid surface. Tribology Letters 7, 2-3 (1999), 147-152.

John W Pritchett. 1978. A numerical model of gas fluidized beds. In AIChE Symposium. 134-148.

P Rajitha, RP Chhabra, NE Sabiri, and Jacques Comiti. 2006. Drag on non-spherical particles in power law non-Newtonian media. International fournal of Mineral Processing 78, 2 (2006), 110-121.

Daniel Ram, Theodore Gast, Chenfanfu Jiang, Craig Schroeder, Alexey Stomakhin, Joseph Teran, and Pirouz Kavehpour. 2015. A material point method for viscoelastic fluids, foams and sponges. In Proceedings of the 14th ACM SIGGRAPH/Eurographics Symposium on Computer Animation. ACM, 157-163.

Bo Ren, Chenfeng Li, Xiao Yan, Ming C Lin, Javier Bonet, and Shi-Min Hu. 2014. Multiplefluid SPH simulation using a mixture model. ACM Transactions on Graphics (TOG) 33, 5 (2014), 171.

Maurice Renaud, Evelyne Mauret, and Rajendra P Chhabra. 2004. Power-law fluid flow over a sphere: Average shear rate and drag coefficient. The Canadian fournal of Chemical Engineering 82, 5 (2004), 1066-1070.

W. Rungjiratananon, Y. Kanamori, and T. Nishita. 2012. Wetting effects in hair simulation. Computer Graphics Forum 31, 7 (2012), 1993-2002.

Witawat Rungjiratananon, Zoltan Szego, Yoshihiro Kanamori, and Tomoyuki Nishita 2008. Real-time Animation of Sand-Water Interaction. Computer Graphics Forum 27, 7 (2008), 1887-1893.

AJC de Saint-Venant. 1871. Theorie du mouvement non permanent des eaux, avec application aux crues des rivieres et a l'introduction de marees dans leurs lits. Comptes rendus des seances de l'Academie des Sciences 36 (1871), 174-154.

Pierre Saramito and Anthony Wachs. 2017. Progress in numerical simulation of yield stress fluid flows. Rheologica Acta 56, 3 (2017), 211-230.

WR Schowalter. 1988. The behavior of complex fluids at solid boundaries. Fournal of Non-Newtonian Fluid Mechanics 29 (1988), 25-36.

Andrew Selle, Michael Lentine, and Ronald Fedkiw. 2008. A mass spring model for hair simulation. ACM Transactions on Graphics (TOG) 27, 3 (2008), 64.

SideFX. 2019. SideFX Houdini. https://www.sidefx.com.

Juan C Simo. 1988. A framework for finite strain elastoplasticity based on maximum plastic dissipation and the multiplicative decomposition: Part I. Continuum for mulation. Computer methods in applied mechanics and engineering 66, 2 (1988), 199-219.

Breannan Smith, Fernando De Goes, and Theodore Kim. 2018. Stable neo-hookean flesh simulation. ACM Transactions on Graphics (TOG) 37, 2 (2018), 12.

Taha Sochi. 2011. Slip at fluid-solid interface. Polymer Reviews 51, 4 (2011), 309-340.

Carlota Soler, Tobias Martin, and Olga Sorkine-Hornung. 2018. Cosserat Rods with Projective Dynamics. Computer Graphics Forum 37, 8 (2018), 137-147.

Jonas Spillmann and Matthias Teschner. 2007. CoRdE: Cosserat rod elements for the dynamic simulation of one-dimensional elastic objects. In Proceedings of the 2007 ACM SIGGRAPH/Eurographics Symposium on Computer animation. Eurographics Association, 63-72.

Jos Stam. 1999. Stable fluids. In Proceedings of the 26th annual conference on Computer graphics and interactive techniques (SIGGRAPH). ACM Press/Addison-Wesley Publishing Co., 121-128.

Alexey Stomakhin, Craig Schroeder, Lawrence Chai, Joseph Teran, and Andrew Selle. 2013. A material point method for snow simulation. ACM Transactions on Graphics (TOG) 32, 4 (2013), 102

Alexey Stomakhin, Craig Schroeder, Chenfanfu Jiang, Lawrence Chai, Joseph Teran, and Andrew Selle. 2014. Augmented MPM for phase-change and varied materials. ACM Transactions on Graphics (TOG) 33, 4 (2014), 138.

Deborah Sulsky, Zhen Chen, and Howard L Schreyer. 1994. A particle method for history-dependent materials. Computer methods in applied mechanics and engineering 118, 1-2 (1994), 179-196

Hervé Tabuteau, Philippe Coussot, and John R de Bruyn. 2007. Drag force on a sphere in steady motion through a yield-stress fluid. Journal of rheology 51, 1 (2007), 125-137.

Andre Pradhana Tampubolon, Theodore Gast, Gergely Klár, Chuyuan Fu, Joseph Teran, Chenfanfu Jiang, and Ken Museth. 2017. Multi-species simulation of porous sand and water mixtures. ACM Transactions on Graphics (TOG) 36, 4 (2017), 105.

Yutaka Tsuji, Toshihiro Kawaguchi, and Toshitsugu Tanaka. 1993. Discrete particle simulation of two-dimensional fluidized bed. Powder technology 77, 1 (1993), 79-87.

Orestis Vantzos, Omri Azencot, Max Wardeztky, Martin Rumpf, and Mirela Ben-Chen 2017. Functional Thin Films on Surfaces. IEEE Transactions on Visualization and Computer Graphics 23, 3 (2017), 1179-1192.

Orestis Vantzos, Saar Raz, and Mirela Ben-Chen. 2018. Real-time Viscous Thin Films. ACM Transactions on Graphics (TOG) 37, 6, Article 281 (Nov. 2018), 10 pages.

Huamin Wang, Gavin Miller, and Greg Turk. 2007. Solving general shallow wave equations on surfaces. In Proceedings of the 2007 ACM SIGGRAPH/Eurographics Symposium on Computer animation (SCA '07). Eurographics Association, 229-238.

Kelly Ward, Florence Bertails, Tae-Yong Kim, Stephen R. Marschner, Marie-Paule Cani, and Ming C. Lin. 2007. A survey on hair modeling: Styling, simulation, and rendering IEEE TVCG 13, 2 (2007), 213-234.
Joshuah Wolper, Yu Fang, Minchen Li, Jiecong Lu, Ming Gao, and Chenfanfu Jiang. 2019. CD-MPM: Continuum Damage Material Point Methods for Dynamic Fracture Animation. ACM Transactions on Graphics (TOG) 38, 4 (2019).

Xiao Yan, Yun-Tao Jiang, Chen-Feng Li, Ralph R Martin, and Shi-Min Hu. 2016. Multiphase sph simulation for interactive fluids and solids. ACM Transactions on Graphics (TOG) 35, 4 (2016), 79.

Tao Yang, Jian Chang, Ming C Lin, Ralph R Martin, Jian J Zhang, and Shi-Min Hu. 2017. A unified particle system framework for multi-phase, multi-material visual simulations. ACM Transactions on Graphics (TOG) 36, 6 (2017), 224.

Tao Yang, Jian Chang, Bo Ren, Ming C Lin, Jian Jun Zhang, and Shi-Min Hu. 2015. Fast multiple-fluid simulation using Helmholtz free energy. ACM Transactions on Graphics (TOG) 34, 6 (2015), 201.

K Yazdchi and Stefan Luding. 2012. Towards unified drag laws for inertial flow through fibrous materials. Chemical Engineering fournal 207 (2012), 35-48.

Thomas Young. 1805. III. An essay on the cohesion of fluids. Philosophical transactions of the royal society of London 95 (1805), 65-87.

Yonghao Yue, Breannan Smith, Christopher Batty, Changxi Zheng, and Eitan Grinspun. 2015. Continuum foam: A material point method for shear-dependent flows. ACM Transactions on Graphics (TOG) 34, 5 (2015), 160.

Yonghao Yue, Breannan Smith, Peter Yichen Chen, Maytee Chantharayukhonthorn, Ken Kamrin, and Eitan Grinspun. 2018. Hybrid Grains: Adaptive Coupling of Discrete and Continuum Simulations of Granular Media. ACM Transactions on Graphics (TOG) 37, 6, Article 283 (Nov. 2018), 19 pages.

Wenqi Zhong, Aibing Yu, Xuejiao Liu, Zhenbo Tong, and Hao Zhang. 2016. DEM/CFDDEM modelling of non-spherical particulate systems: theoretical developments and applications. Powder Technology 302 (2016), 108-152.

ZY Zhou, SB Kuang, KW Chu, and AB Yu. 2010. Discrete particle simulation of particlefluid flow: model formulations and their applicability. Fournal of Fluid Mechanics 661 (2010), 482-510.

Bo Zhu, Minjae Lee, Ed Quigley, and Ronald Fedkiw. 2015. Codimensional nonNewtonian fluids. ACM Transactions on Graphics (TOG) 34, 4 (2015), 115.

Yongning Zhu and Robert Bridson. 2005. Animating sand as a fluid. ACM Transactions on Graphics (TOG) 24, 3 (2005), 965-972. 